\title{
Optical and Ultraviolet Monitoring of the Black Hole X-ray Binary MAXI J1820+070/ASASSN-18ey for 18 Months
}

Hanna Sai, ${ }^{1 \star}$ Xiaofeng Wang, ${ }^{1,2} \dagger$ Jianfeng Wu, ${ }^{3}$ Jie Lin, ${ }^{1}$ Hua Feng, ${ }^{4,5}$ Tianmeng Zhang, ${ }^{6,7}$ Wenxiong Li, ${ }^{1}$ Jujia Zhang, $, 8,9,10$ Jun Mo, ${ }^{1}$ Tianrui Sun, ${ }^{11,12,13}$ Shuhrat A. Ehgamberdiev, ${ }^{14}$ Davron Mirzaqulov, ${ }^{14}$ Liming Rui, ${ }^{1}$ Weili Lin, ${ }^{1}$ Xulin Zhao, ${ }^{15}$ Han Lin, ${ }^{1}$ Jicheng Zhang, ${ }^{1}$ Xinghan Zhang, ${ }^{1}$ Yong Zhao, ${ }^{6,16}$ Xue Li, ${ }^{1}$ Danfeng Xiang, ${ }^{1}$ Lingzhi Wang, ${ }^{17,18}$ Chengyuan W

${ }^{1}$ Physics Department and Tsinghua Center for Astrophysics (THCA), Tsinghua University, Beijing, 100084, China

${ }^{2}$ Beijing Planetarium, Beijing Academy of Science and Technology, Beijing, 100044, China

${ }^{3}$ Department of Astronomy Xiamen University (Haiyun Campus), Siming District, Xiamen, Fujian, 361005, China

${ }^{4}$ Department of Astronomy, Tsinghua University, Beijing, 100084, China.

${ }^{5}$ Department of Engineering Physics, Tsinghua University, Beijing, 100084, China.

${ }^{6}$ Key Laboratory of Optical Astronomy, National Astronomical Observatories, Chinese Academy of Sciences, Beijing, 100101, China

${ }^{7}$ School of Astronomy and Space Science, University of Chinese Academy of Sciences, 101408, Beijing

${ }^{8}$ Yunnan Observatories (YNAO), Chinese Academy of Sciences, Kunming 650216, China

${ }^{9}$ Key Laboratory for the Structure and Evolution of Celestial Objects, Chinese Academy of Sciences, Kunming 650216, China

${ }^{10}$ Center for Astronomical Mega-Science, Chinese Academy of Sciences, 20A Datun Road, Chaoyang District, Beijing, 100012, China

${ }^{11}$ Purple Mountain Observatory, Chinese Academy of Sciences, Nanjing 210008, China

${ }^{12}$ Chinese Center for Antarctic Astronomy, Nanjing 210008, China

${ }^{13}$ School of Astronomy and Space Science, University of Science and Technology of China, Hefei 230026, China

${ }^{14}$ Ulugh Beg Astronomical Institute, Uzbekistan Academy of Sciences, Uzbekistan, Tashkent, 100052, Uzbekistan

${ }^{15}$ School of Science, Tianjin University of Technology, Tianjin, 300384, China

${ }^{16}$ University of Chinese Academy of Sciences, Beijing 100049, China

${ }^{17}$ National Astronomical Observatory of China, Chinese Academy of Sciences, Beijing, 100012, China

${ }_{18}$ Chinese Academy of Sciences South America Center for Astronomy, National Astronomical Observatories, CAS, Beijing 100101, China

Accepted XXX. Received YYY; in original form ZZZ

\begin{abstract}
MAXI J1820+070 is a low-mass black hole X-ray binary system with high luminosity in both optical and X-ray bands during the outburst periods. We present extensive photometry in X-ray, ultraviolet, and optical bands, as well as densely-cadenced optical spectra, covering the phase from the beginning of optical outburst to $\sim 550$ days. During the rebrightening process, the optical emission preceded the X-ray by 20.80 \pm 2.85 days. The spectra are characterized by blue continua and emission features of Balmer series, He I, He II lines and broad Bowen blend. The pseudo equivalent width $(\mathrm{pEW})$ of emission lines are found to show anticorrelations with the X-ray flux measured at comparable phases, which is due to the increased suppression by the optical continuum. At around the X-ray peak, the full width at half maximums (FWHMs) of $\mathrm{H}_{\beta}$ and $\mathrm{He}$ II $\lambda 4686$ tend to stabilize at 19.4 Angstrom and 21.8 Angstrom, which corresponds to the line forming region at a radius of 1.7 and $1.3 R_{\odot}$ within the disk. We further analyzed the absolute fluxes of the lines and found that the fluxes of $\mathrm{H}_{\beta}$ and He II $\lambda 4686$ show positive correlations with the X-ray flux, favoring that the irradiation model is responsible for the optical emission. However, the fact that X-ray emission experiences a dramatic flux drop at $\mathrm{t} \sim 200$ days after the outburst, while the optical flux only shows little variations suggests that additional energy such as viscous energy may contribute to the optical radiation in addition to the X-ray irradiation.
\end{abstract}

Key words: accretion, accretion discs - stars: black holes - X-rays: binaries 


\section{INTRODUCTION}

Low-mass X-ray binaries (LMXBs) are close binary systems consisting of a compact object and a low-mass companion star having filled its Roche lobe. The mass-donor companion can be a main-sequence star, a subgiant, or a white dwarf (WD), while the compact object can be a black hole (BH) or a neutron star (NS). Materials of the donor star transfer to the compact object through the $L 1$ Lagrangian point, forming a surrounding accretion disc (Charles \& Coe 2006; Gu et al. 2019). LMXBs often stay for years or decades in a state of quiescence with X-ray luminosities of $\sim 10^{29}-10^{33.5} \mathrm{erg}$ $\mathrm{s}^{-1}$ before turning into outbursts (Remillard \& McClintock 2006). The mass of a BH in an X-ray binary can be determined based on the periodic variations inferred from the spectroscopic and photometric observations in quiescence (e.g.,Casares \& Jonker 2014; Wu et al. 2015, 2016). During the outburst, the luminosity of these LMXBs can increase by many orders of magnitudes relative to its quiescent state (Chen et al. 1997). The outbursts can be explained by instability of accretion discs surrounding the compact object (Osaki 1995; Lasota 2001; Dubus et al. 2001), which is also called the "thermal-viscous disc instability model" (DIM). Some LMXB transients can experience several outbursts, and those with higher mass transfer rate tend to have higher outburst frequencies (Lin et al. 2019).

According to the irradiated disk model, the optical and ultraviolet (UV) -band emissions are believed to arise from reprocessing of the X-ray emission (Rykoff et al. 2007; Gierliński et al. 2008, 2009). In this scenario, the temperature structure of the outer disc will be altered by the irradiated inner disk and Compton tail, leading to production of the observed optical/UV emission (Gierliński et al. 2009). The optical spectra of LMXBs often contain emission lines, such as the Balmer series. The emission lines are believed to originate from rotating accretion flow of the disc, which is ionized by irradiation from a central high-luminosity X-ray source. Note, however, that there are other arguments about the origin of the optical radiation of LMXB transients, such as the jet/corona (Eikenberry et al. 1998; Markoff et al. 2005; Russell et al. 2006) or the atmosphere of an optically thick disc in bright/soft X-ray states (Wu et al. 2001, 2002). Moreover, the $\mathrm{H}_{\alpha}$ emission line could also originate in a dense outflow in low/hard X-ray states (Wu et al. 2001). At low-luminosity state, the ionizing source is usually considered to be viscous heating of the disc (van Paradijs \& McClintock 1994; Fender et al. 2009).

The outburst of the LMXB system, MAXI J1820+070 (ASASSN-18ey), was initially discovered as an optical transient ASASSN-18ey on UT Mar.06.58 2018 by the All Sky Automated Survey for SuperNovae (ASAS-SN; Shappee et al. 2014; Kochanek et al. 2017) at R.A. $=18^{h} 20^{m} 21^{s} .9$, dec. $=+07^{\circ} 11^{\prime} 07^{\prime \prime} .3(\mathrm{~J} 2000)^{1}$. About one week later, the Monitor of All-sky X-ray Image (MAXI; Matsuoka et al. 2009) Gas Slit Camera (Mihara et al. 2011) nova alert system detected a bright X-ray transient at the same location with a flux of $32 \pm 9 \mathrm{mCrab}$ in $4-10 \mathrm{keV}$ (Kawamuro et al. 2018), which was designated as MAXI J1820+070. Multiwavelength follow-up observations suggested that MAXI $\mathrm{J} 1820+070$ is a BH LMXB (e.g., Baglio et al. 2018; Homan

1 http://www.astronomy.ohio-state.edu/asassn/transients.html et al. 2018; Tucker et al. 2018). The distance to this system is estimated as $3.46_{-1.03}^{+2.18} \mathrm{kpc}$ according to the Gaia DR2 parallax data (Gandhi et al. 2019). Atri et al. (2020) provided a consistent and more precise measurement on the distance to MAXI J1820+070 as $2.96 \pm 0.33 \mathrm{kpc}$ using the parallax obtained from radio interferometry.

The X-ray and optical monitoring of this system showed that there is a time lag of $7.20 \pm 0.97$ days between the outbursts detected in optical and X-ray bands for MAXI $\mathrm{J} 1820+070$ in March, 2018 (Tucker et al. 2018). This delay suggests that the thermal instability initially triggered an outburst in the thermal-viscous disc. The X-ray and optical monitoring of this system suggests that the state transition is not only related to the mass accretion rate (Shidatsu et al. 2019). They proposed that the jet contributed to the optical emission in the low/hard state, whereas the outer disc emission dominated the optical flux in high/soft state. Polarization observations also favored for the existence of jet or hot flow for MAXI J1820+070 (Veledina et al. 2019). Dynamical modeling of MAXI J1820+070 system was carried out by Torres et al. (2019). They confirmed the property of stellarmass BH for the compact primary of MAXI J1820+070, with its mass function as $f(M)=5.18 \pm 0.15 M_{\odot}$ and an orbital period of $0.68549 \pm 0.00001$ days. Muñoz-Darias et al. (2019) detected P-Cygni profiles and broad wings in He I $\lambda 5876$ and $\mathrm{H}_{\alpha}$ emission lines in the high-resolution optical spectra of MAXI J1820+070 taken during low/hard states, suggesting the existence of accretion disc wind.

In this paper, we report extensive follow-up observations of MAXI J1820+070 in optical, UV, and X-ray bands for about 18 months, and present its observational properties. The relations between optical/UV and X-ray features have been analyzed, with an attempt to better constrain the radiation physics for this $\mathrm{BH}$ binary system. The paper is organized as follows: in Section 2, the observations and data reductions are described. Section 3 presents the light/color curves, and Section 4 presents the spectral evolution. In Section 5, we discuss the properties of MAXI J1820+070, including spectral energy distribution (SED) and the correlation between optical and X-ray properties. We summarize our results in Section 6.

\section{OBSERVATIONS AND DATA REDUCTION}

After the discovery of MAXI J1820+070, we started a longterm spectroscopic and photometric monitoring campaign in optical bands. The follow-up photometric observations started on March 20, 2018, using the 0.8-m Tsinghua-NAOC Telescope (TNT) (Huang et al. 2012; see Figure 1 for a sample image) and the Yaoan High Precision Telescope in China, as well as the AZT-22 1.5-m telescope (hereafter AZT) at the Maidanak Astronomical Observatory in Uzbekistan (Ehgamberdiev 2018). We use standard IRAF routines to pre-process all CCD images, which include bias subtraction, flat fielding, and the removal of cosmic rays. Point-spreadfunction (PSF) photometry was performed for both the object and the reference stars using the pipeline Zuruphot developed for automatic photometry of TNT (Mo et al. in prep.). The instrumental magnitudes were then converted into those of the Johnsons $B V$ (Johnson et al. 1966) and Sloan Digital Sky Survey (SDSS) gri-band system (Fukugita 
et al. 1996). Table A1 lists the standard BV- and gri-band magnitudes of the comparison stars, which are also marked in Figure 1. MAXI J1820+070 was also observed by the Ultraviolet/Optical Telescope (UVOT; Roming et al. 2005) onboard the Neil Gehrels Swift Observatory (Swift; Gehrels et al. 2004) in three UV (uvw2, uvm2 and uvw1) and three optical filters $(u, b$ and $v)$. The final calibrated magnitudes in the Swift filters are presented in Table A2 and Table A3, respectively. The Swift images of MAXI J1820+070 were reduced using the HEASOFT ${ }^{2}$ with the latest Swift calibration database ${ }^{3}$.

We also analyzed the Swift/XRT observations of MAXI J1820+070 obtained during the period from MJD 58189 to MJD 58591. These observations were obtained in both window timing (WT) and photon count (PC) modes. All of the XRT data were first reduced by running the xrtpipeline task of HEASOFT (version 6.19). Since the count rate of MAXI $\mathrm{J} 1820+070$ can reach beyond $500 \mathrm{cps}$, the source region suffers serious pile-up effect. To avoid this effect, we extract the source photons using an annular region. The outer radius is set as 40 pixels, and the inner radius is adjustable according to the count rates. In the WT mode observations, the inner radius is set as RATE/100 pixels (RATE stands for the count rate). In the $\mathrm{PC}$ mode observations, the inner radius is fixed as 2 pixels if the count rate is higher than $0.5 \mathrm{cps}$; otherwise, the inner radius is set to zero. The background photons were estimated from an annular region with an outer radius of 120 pixels and an inner radius of 60 pixels. The energy spectra were extracted by the XSELECT package and grouped into bins with a minimum of 20 photons. We adopted response matrix files (RMFs) from the Swift/XRT calibration database (CALDB) and created ancillary response files (ARFs) from exposure maps using the xrtmkarf task. The XRT spectra between 0.6 and 10 $\mathrm{keV}$ were fitted with a model composed of a power-law component (the powerlaw model) plus a multi-black body component (the diskbb model). The power-law component accounts for the comptonized X-ray emission, and the multiblack body component represents the thermal X-ray emission from the surface of the accretion disc, while the X-ray absorption is characterized by the wabs model. During the phase from 336 to 371 days after the initial detection, the $\mathrm{X}$-ray count rate is quite low so we use a single power-law model to estimate the flux (Kirsch et al. 2005).

A total of 66 spectra were obtained for MAXI J1820+070 with the Lijiang 2.4-m telescope (+YFOSC) and Xinglong 2.16-m telescope (+BFOSC and +OMR) (Zhang et al. 2016), covering the phases from +11.9 days to +385.3 days since the discovery. A log of the spectra is given in Table A4. We reduced all the spectra using standard IRAF routine. Flux calibration of the spectra was performed with spectrophotometric standard stars taken on the same nights. The spectra were corrected for atmospheric extinction using the extinction curves of local observatories; and the telluric lines were removed from the spectra.

2 HEASOFT, the High Energy Astrophysics Software https://www.swift.ac.uk/analysis/software.php

3 https://heasarc.gsfc.nasa.gov/docs/heasarc/caldb/swift/

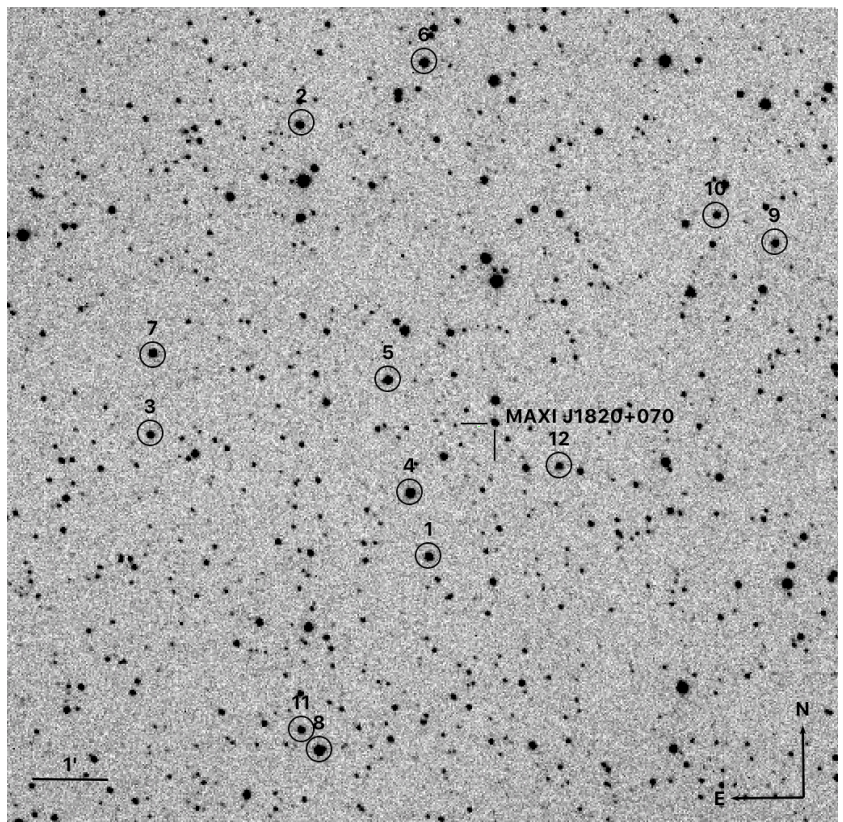

Figure 1. Image of MAXI J1820+070/ASASSN-18ey, taken with the Tsinghua-NAOC $0.8-\mathrm{m}$ Telescope (TNT). North is up and east is to the left. MAXI J1820+070 is indicated by the solid thick sticks. The reference stars are labeled with black circles.

\section{LIGHT CURVES}

Figure 2 shows the optical and UV light curves of MAXI $\mathrm{J} 1820+070$, covering the phase from +6.92 to +536.67 days relative to the first detection of optical outburst. For the TNT observations, the first gap appeared between July and August, 2018, covering the phase from +114 to +185 days, is due to the maintenance of the telescope in the summer season, while the second gap, seen during the phase from $\mathrm{t} \sim+258$ to +372 days, is because that the source moved close to the sun. To complement the sampling of the light curves, we also collected the $B$-band data from the American Association of Variable Star Observers (AAVSO).

At $\mathrm{t} \sim+100$ days and 210 days after outburst, MAXI $\mathrm{J} 1820+070$ is found to exhibit two rebrightening behaviors. However, it did not show quiescence between these two epochs. The first rebrightening occurred before the transition from low/hard to high/soft states, while the second one occurred shortly after the transition from the high/soft to low/hard states. By $\mathrm{t} \sim+360$ days, this system faded gradually towards its quiescent state (Russell et al. 2019b). At $\mathrm{t} \sim+370$ days, however, this system is found to experience a post-outburst "rebrightening" in both optical and X-ray bands (Ulowetz et al. 2019; Bahramian et al. 2019), and reached a B-band peak of $14.17 \mathrm{mag}$ at $\mathrm{t} \sim+380$ days. This corresponds to an absolute magnitude of $1.47 \mathrm{mag}$ in $\mathrm{B}$ band using the Gaia distance (Gandhi et al. 2019). After that, MAXI J1820+070 tended to decrease in both X-ray and optical bands and entered into the quiescent state based on the X-ray flux reported by Swift and NuSTAR (Vozza et al. 2019; Tomsick \& Homan 2019). At $\mathrm{t} \sim+507$ days, MAXI $\mathrm{J} 1820+070$ was found to rebrighten again in opitcal (see Figure 2) and other bands including radio, near-infrared and 
X-ray bands (see Hambsch et al. 2019, Xu et al. 2019, Bright et al. 2019 and Hankins et al. 2019).

\section{OPTICAL SPECTRA}

\subsection{Spectral Evolution}

Figure 3 shows the spectral evolution of MAXI J1820+070. The spectra are characterized by blue continua superimposed with $\mathrm{H}_{\alpha} \lambda 6563, \mathrm{H}_{\beta} \lambda 4861, \mathrm{H}_{\gamma} \lambda 4341$, He I $\lambda 5876$, He I $\lambda 6678$, He II $\lambda 4686$ lines and Bowen blend. The detailed evolution of the main spectral features, i.e., $\mathrm{H}_{\alpha}, \mathrm{H}_{\beta}$, $\mathrm{H}_{\gamma}$, and $\mathrm{He} \mathrm{I} \lambda 5876$ are shown in Figure 4 . The profiles of these features all show some degrees of temporal evolution, with some having only a single Gaussian component while others showing asymmetric line profiles, or double-peaked emission, as shown in Figure 5. Using the Bayesian information criteria (BIC), a measure of the relative quality for a fit in model-selection (Schwarz 1978), we find that double Gaussian model $(\mathrm{BIC}=-548.8)$ works better than the single Gaussian model $(\mathrm{BIC}=-445.8)$ for $\mathrm{H}_{\alpha}$ line at some phases (i.e., $\mathrm{t}=+39.78$ day). However, in other phases (i.e., $\mathrm{t}=+113.63$ day), the amplitudes of two sub-components of double Gaussian model differ so greatly that the weaker component is negligible. In this case, the single Gaussian model is still a better fitting model for such profiles. The single/double-peaked profile transition of emission lines was previously reported in many BH X-ray binaries (Fender et al. 2009; Mata Sánchez et al. 2018). A double-peaked line profile should originate from a rotating flow, i.e., the geometrically thin accretion discs (Meyer 1986), while winds from the accretion disc, driven by radiation pressure, tend to produce single-peaked lines (Murray \& Chiang 1996). However, the asymmetric single-line profile in our spectra can be formed by double Gaussian components which blended due to lower spectral resolution. Adding to the complexity, an apparently double-peaked emission line can be also produced from an intrinsically single-peaked profile with a central absorption (Fender et al. 2009). Given the above uncertainties in applying the double gaussian fit, we thus adopted the single gaussian fit in the following analysis of the main spectral features.

\subsection{Balmer Series}

In Figure 6, we present the temporal evolution of parameters measured from the Balmer emission lines. The local pseudocontinuum is determined via a linear fit to both sides of the feature; and repeated visual inspections are required for reducing the noise (Zhao et al. 2016). The pEW is calculated with the equation below:

$\mathrm{pEW}=\int\left(F_{\mathrm{o}}-F_{\mathrm{c}}\right) / F_{\mathrm{c}} d \lambda$

where $F_{\mathrm{o}}$ is the observed flux and $F_{\mathrm{c}}$ is the flux of the local pseudo-continuum.

Note that the $\mathrm{H}_{\alpha}$ line shows a clear trend of strengthening within $t \sim 300$ days from the initial detection of optical outburst, while its strength tends to decrease after $\mathrm{t} \sim 370$ days. The $\mathrm{pEW}$ of $\mathrm{H}_{\beta}$ line holds a similar evolutionary trend as $\mathrm{H}_{\alpha}$. Such an evolution pattern in Balmer lines can be explained with the change in optical depth as discussed in
Williams (1980) and Fender et al. (2009). The spectrum of LMXB consists of a thermal continuum produced by inner hot, optically thick region of the disk, and emission lines formed in the outer cool region where the continuum is optically thin. During the rebrightening phase, the outer disc becomes hot as the X-ray irradiation gets stronger, and this makes the continuum become optically thick. The $\mathrm{pEW}$ of the emission lines depends on the relative size of the regions in the disk that are optically thin and thick in the continuum according to Williams (1980). Thus, the portion which is optically thick in the continuum increases during the rebrightening and this leads to decrease in pEW of optical lines from the outer region. The growth trend within $\sim 300$ days can be also explained by this model. As the outer disk gets colder, an optically thin continuum forms at this stage and it leads to the increase in the pEWs of optical hydrogen emission lines.

To further examine the origin of the variation in $\mathrm{H}_{\alpha}$ emission, we show in Figure 7 the correlation between the pEW of $\mathrm{H}_{\alpha}$ and the $r$ - band magnitude, which can characterize the strength of the continuum. The trend indicates that the $\mathrm{pEW}$ of $\mathrm{H}_{\alpha}$ emission increases when the system becomes faint in optical, and vice versa. It is consistent with the optical depth explanation of the evolution pattern in Balmer lines.

\subsection{He I $\lambda 5876$ Feature}

He I $\lambda 5876$ is found to show persistent P-Cygni profiles in the spectra of MAXI J1820+070 taken during the Xray low/hard state, as similarly reported by Muñoz-Darias et al. (2019). Similar P-Cygni profile was also seen in V404 Cygni and other BH LMXBs (Mata Sánchez et al. 2018; Casares et al. 2019), where the continuous presence of PCygni profiles were proposed to result from an expanding outflow formed during the outburst. One can notice that there is a prominent absorption feature on the red side of He I $\lambda 5876$, which is likely due to the Galactic Na I D absorption doublet. The evolution of He I $\lambda 5876$ emission line parameters is plotted in Figure 6, where one can see that the $\mathrm{pEW}$ follows a similar evolution trend as seen in $\mathrm{H}_{\beta}$.

\section{DISCUSSION}

\subsection{SED and Temperature}

To examine the radiation characteristics of MAXI J1820+070, we constructed the spectral energy distribution (SED) with the Swift/UVOT, ground-based $B, g, V, r$, and $i$-band photometry to derive the temperature and radius of the photosphere, as shown in Figure 8. During the outburst phases, the optical and UV emissions from the companion star are negligible compared to those from the accretion disc (and possibly jets). The corresponding SEDs, corrected for the galactic extinction, is shown in Figure 8. It can be seen that in general the SEDs can not be well described by a single blackbody model, with the deviations mostly coming from the UV bands. This suggests that the emission regions of the optical and UV bands have different temperatures, i.e., a multi-temperature disc. However, fitting to the optical and UV bands separately would not provide useful con- 


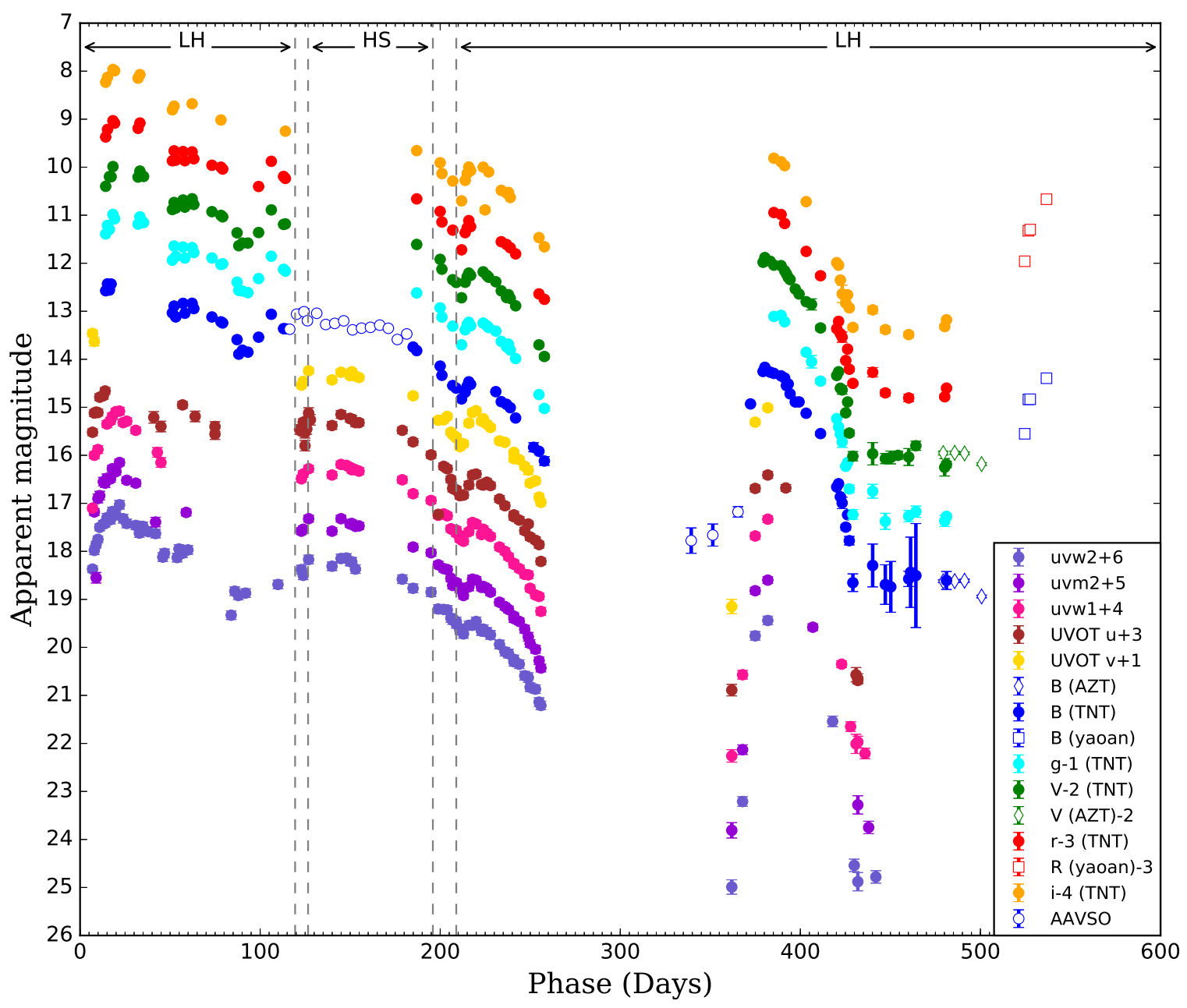

Figure 2. The optical and UV light curves obtained for MAXI J1820+070, lasting for about 550 days after the initial optical outburst (UT March 06.582018 = MJD 58184.08). Different colors represent different bands, including the Swift uvw2, uvm2, uvw1, UVOT u, UVOT $v, B, g, V, r, R, i$, and $I$ bands. The light curves of different bands have been vertically shifted for better display. The arrow labels with 'LH' and 'HS' on the top represent the low/hard and high/soft state periods, respectively. The two narrow gaps between the LH and HS are intermediate-state periods (Shidatsu et al. 2019).

straints on different radiation zones because of limited data available for analysis. Contributions from the jets may also play a role, though they usually peak in the near-infrared bands (e.g., Corbel \& Fender 2002; Russell et al. 2006).

Although the accretion disk may have multitemperature components, the observed SED can still be reasonably fit by a simple blackbody model (Tucker et al. 2018), as shown in Figure 9. One can see that the blackbody temperature inferred from the multicolor photometry shows a rather complicated evolution, with an initial rapid increase from $\sim 17,000 \mathrm{~K}$ to $23,000 \mathrm{~K}$, followed by a sudden drop to $15,000 \mathrm{~K}$ at $\mathrm{t} \sim 250$ days. After a possible plateau evolution during $\mathrm{t} \sim 100-200$ days, the temperature then suffered another dramatic decline, i.e. from $15,000 \mathrm{~K}$ at $\mathrm{t} \sim 250$ days to $8,000 \mathrm{~K}$ at $\mathrm{t} \sim 450$ days.
During the period from $\mathrm{t} \sim 15$ to $\sim 80$ days after the initial outburst, MAXI J1820+070 appeared to become gradually hotter as its photosphere receded, which is consistent with the results from Tucker et al. (2018). From t $\sim 180$ to $\sim 260$ days, the optical and UV emission region cooled down. This is consistent with the Balmer series and He I emission lines becoming stronger during similar phases (see Section 4). After $\mathrm{t} \sim 380$ days, MAXI J1820+070 experienced a non-adiabatic evolution process, with the radiative radius becoming smaller while the temperature of the accretion disc keeping decreasing. Such a temperature decrease is consistent with the redwards evolution seen in the color curve (see Section 3 and Figure 10).

The $B-V, g-r$, and $r-i$ color curves of MAXI J1820+070, and the corresponding Spearman's rank corre- 

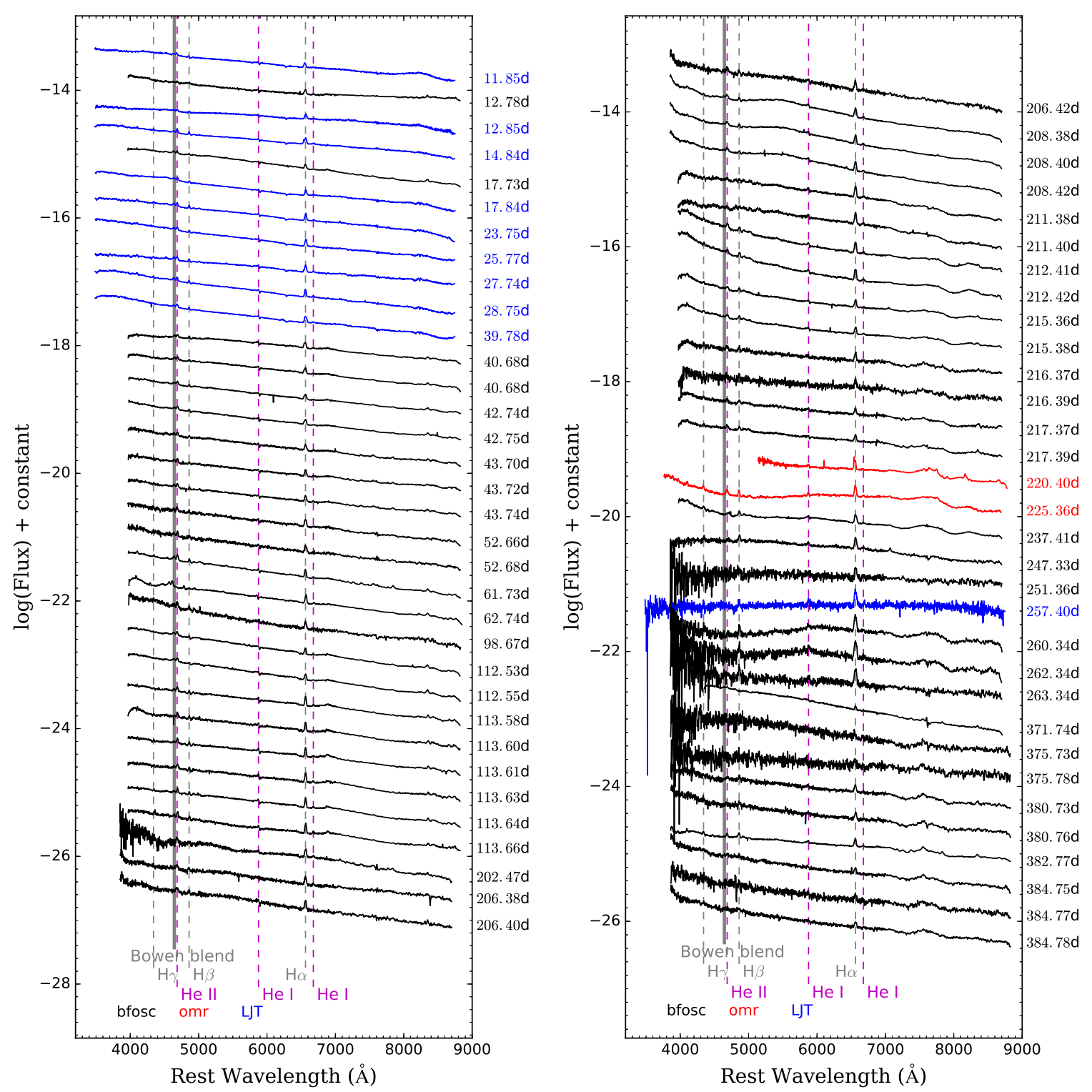

Figure 3. Spectral evolution of MAXI J1820+070. The continuums of the spectra have been flux-calibrated by the photometry obtained at similar phases. The spectra have been shifted vertically for better display. The epoch on the right side of each spectrum represents the phase in days after the initial optical outburst. The Balmer series (including $\mathrm{H}_{\alpha}, \mathrm{H}_{\beta}$, and $\mathrm{H}_{\gamma}$ here) and the helium emission lines are marked by grey and magenta dashed lines, respectively. The Bowen blend feature is labeled by the grey vertical bar in each panel. Different colors of the spectra represent different spectroscopic instruments (i.e., XLT+bfosc, XLT+omr or LJT+YFOSC), which are shown at the bottom of the plot.

lation coefficient with phases are shown in Figure 10. The positive correlations indicate the post-outburst color tends to become progressively redder with a statistical significance of $\sim 4.5 \sigma$. This means the overall temperature of this system gradually decreased with time. However, the real physical process may be more complicated. There are some factors that cause the deviation from single blackbody, such as temperature difference in different regions of accretion disk and changes in optical depth.

\subsection{Optical and X-ray Relation}

As mentioned in Section 1, MAXI J1820 +070 is very bright in both optical and X-ray bands during the outburst. This gives us an opportunity to study the correlation between emission features in optical and the X-ray luminosity and put constraints on the physics of $\mathrm{BH}$ accretion.

In Figure 11, we plot the $\mathrm{pEW}$ of $\mathrm{H}_{\alpha}$ emission as a function of X-ray luminosity inferred for MAXI J1820+070 at comparable phases. We adopted the average value for the pEW of $\mathrm{H}_{\alpha}$ line and X-ray luminosity when multiple obser- 


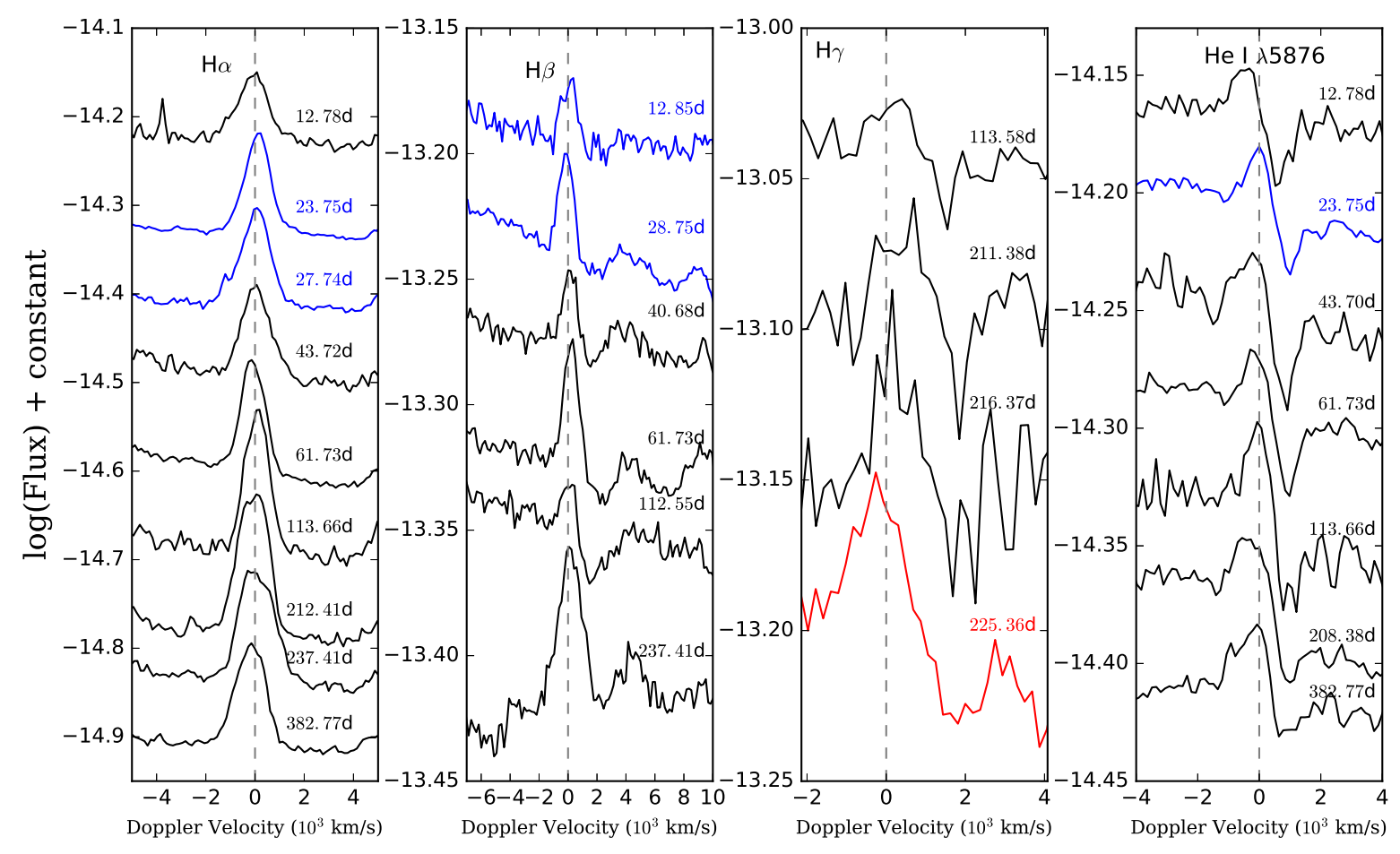

Figure 4. The $\mathrm{H}_{\alpha}, \mathrm{H}_{\beta}, \mathrm{H}_{\gamma}$, and He I $\lambda 5876$ lines of MAXI J1820+070 in velocity space. The colors represent the spectra obtained with the instruments marked in Figure 3.

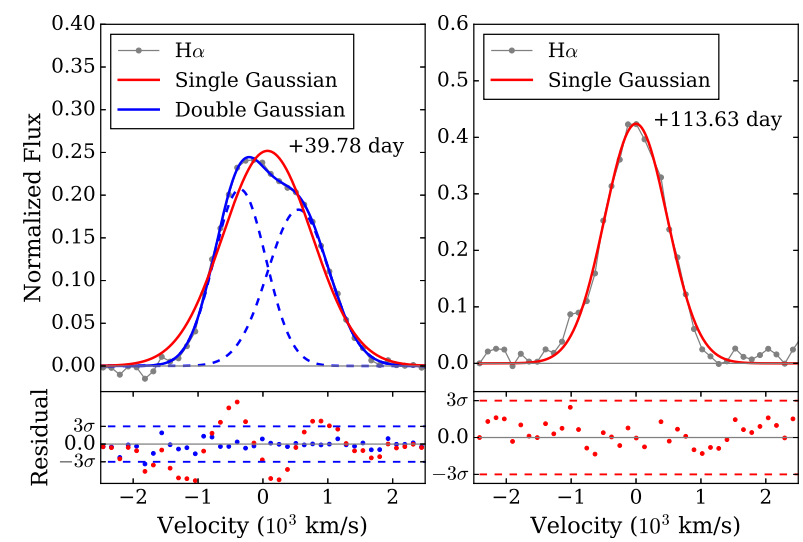

Figure 5. The $\mathrm{H}_{\alpha}$ line profile in two spectra of MAXI J1820+070, displayed in velocity space. Left panel: the $\mathrm{H}_{\alpha}$ line profile in the $\mathrm{t}=+39.78$ day spectrum. The red solid curve represents the fit curve using a single Gaussian function, while the blue solid line is the double Gaussian fit with two sub-components labeled by blue dashed lines. Right panel: the same case as in the left panel but for the $\mathrm{t}=+113.63$ day spectrum. The lower panels show the residuals of the observed line profiles relative to the best-fit profiles.

vations are available on the same day. For MAXI J1820+070, most of these observations were obtained in the low/hard states. An anticorrelation can be seen between X-ray lumi-

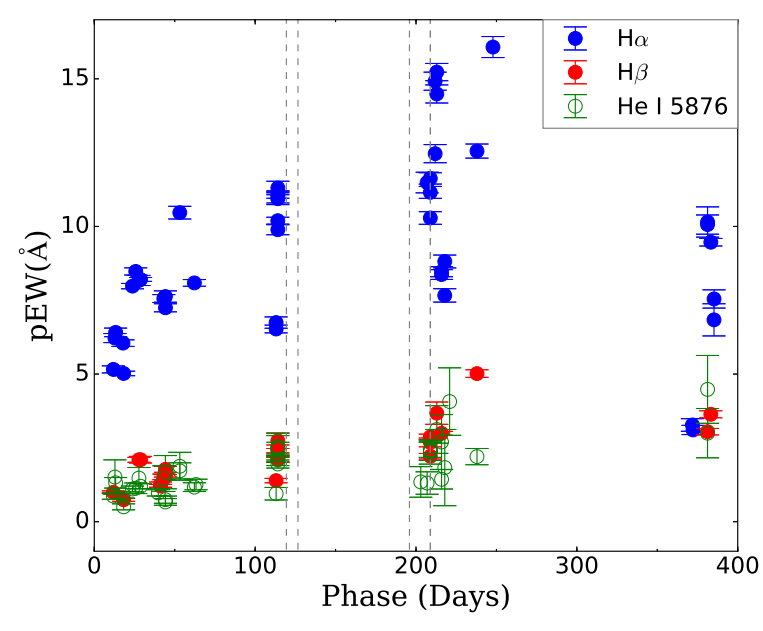

Figure 6. The pseudo equivalent width (pEW) evolution of the Balmer series and He I $\lambda 5876$ in the spectra of MAXI J1820+070. All the lines were fit by single gaussian profiles. We use different colors to represent different spectral lines.

nosity and the corresponding pEW of $\mathrm{H}_{\alpha}$ with a confidence level of $99.1 \%(2.64 \sigma)$. Based on a sample of six BH X-ray binaries, Fender et al. (2009) reported an anticorrelation between $\mathrm{pEW}$ of $\mathrm{H}_{\alpha}$ emission line and X-ray luminosity, which 


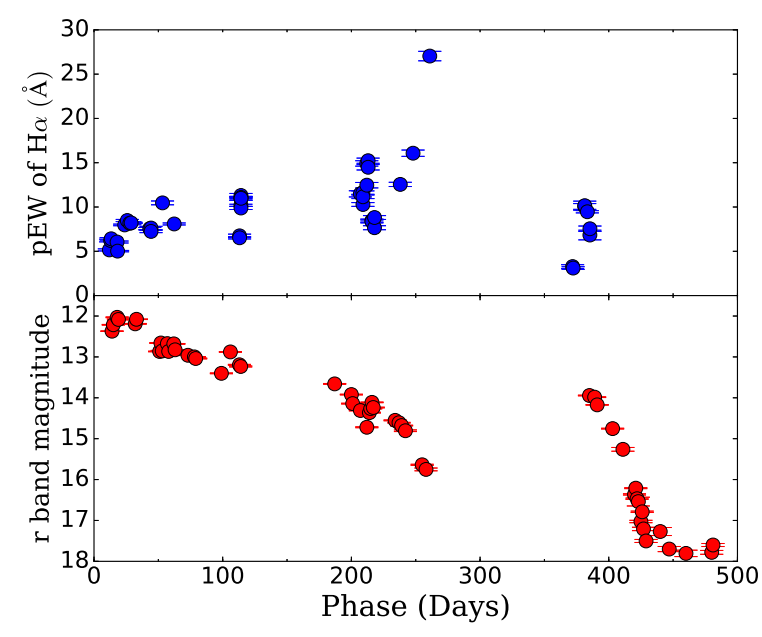

Figure 7. The pseudo equivalent width evolution of $\mathrm{H}_{\alpha}$ (top) and the $r$ - band magnitude (bottom) measured for MAXI J1820+070. The time in days from the initial optical outburst are shown along the bottom axis.

is $\mathrm{pEW} \propto L_{x}^{-0.18 \pm 0.06}$. Applying a power-law model to fit the observations of MAXI J1820+070, we get

$\mathrm{pEW}=10^{4.3 \pm 1.1} L_{x}^{-0.092 \pm 0.030}$

Note that this fit excludes the data point taken on $t \sim+372$ days. Inspecting the X-ray and optical/UV light curves shown in Figure 12, one can see that at t $\sim 372$ days, the optical and X-ray emission of MAXI J18020+070 was still in a rapid rising phase, and the BH binary system is likely in a transition state. This indicates that the anticorrelation between X-ray luminosity and $\mathrm{H}_{\alpha}$ emission holds in the outburst phase but not in the transition phase. Thus the radiation mechanisms of the lines and disks during the transition phase are not consistent with that of the post-outburst phase.

As the measured pEW suffered from the effect of continuum flux, we further examine the relations between the absolute flux of lines such as $\mathrm{H}_{\alpha}, \mathrm{H}_{\beta}$, He II $\lambda 4686$ and 2-10 $\mathrm{keV} \mathrm{X}$-ray flux, as shown in Figure 13. We find that the absolute fluxes of these lines show positive correlations with the $\mathrm{X}$-ray radiation. We did not examine the correlation for He I $\lambda 5876$ due to its possible blending with the $\mathrm{Na}$ I doublet. In comparison, the He II $\lambda 4686$ line seems to show the strongest correlation with the X-ray flux, because He II has a higher ionization energy (i.e., $54.4 \mathrm{eV}$ ) and can only be excited by X-ray photons (Leloudas et al. 2019).

Because the FWHM of $\mathrm{H}_{\beta}$ and He II $\lambda 4686$ lines can be used to determine the outer edge of the accretion disk, we further examined their correlations with X-ray emission in lower-panel of Figure 13. We found that with the increase of $\mathrm{X}$-ray flux, both of these two lines tend to get weaker and then stabilize at $1200 \mathrm{~km} \mathrm{~s}^{-1}$ and $1400 \mathrm{~km} \mathrm{~s}^{-1}$, respectively. Based on the fact that the gas motion in accretion disk can be approximated to follow the Kepler motion, its velocity can be determined by $v=\sqrt{3 /(8 \ln 2)}$ FWHM according to the analysis by Netzer (2013). The corresponding radius of the gas motion in the MAXI J1820+070 system can be estimated as $1.71 \pm 0.13 R_{\odot}$ from $\mathrm{H}_{\beta}$ and $1.25 \pm 0.11 R_{\odot}$ from
He II $\lambda 4686$ line, respectively. We find that both of these two values are less than the typical radius of the accretion disk surrounding the stellar-mass BH (Lasota 2001), which equals to the $90 \%$ of effective Roche radius $R_{L}=2.46 R_{\odot}$, identified by the relevant parameters obtained by Torres et al. (2019). The larger radius inferred from $\mathrm{H}_{\beta}$ relative to $\mathrm{He}$ II $\lambda 4686$ indicates that the formation region of the former line can extend to the outer side of the accretion disc. This is due to the fact that He II $\lambda 4686$ has a higher excitation energy than $\mathrm{H}_{\beta}$. On the outer part of the accretion disk with lower temperature, only $\mathrm{H}_{\beta}$ can be excited. Note, however, that $\mathrm{H}_{\alpha}$ did not show such a similar relationship with X-ray flux. This suggests that the formation region of $\mathrm{H}_{\alpha}$ line was not confined to the accretion disk, but also included the outflow, as indicated by the P-cygni profile of $\mathrm{H}_{\alpha}$ from Muñoz-Darias et al. (2019).

The thermal-viscous DIM describes that, in quiescence, the accretion disc is filled up with matter, until at some radius, its temperature reaches the critical value that ionizes the hydrogen to trigger an outburst (Lasota 2001; Russell et al. 2019a). This outburst can be triggered within the inner part of the accretion disc, and then propagate outwards, which is called as "inside-out" outburst (Lasota 2001). However, in other LMXBs, the triggering process may start at the outer disc and then propagates inwards to the inner region, which is called as "outside-in" outburst (Shahbaz et al. 1998). Optical emission is usually produced by the outer disc, while the X-ray emission can be created on the viscous timescale when the inner disc is filled in. Thus detecting the time lag between different bands helps determine where the instability is triggered in the accretion disk.

Based on the method introduced in Tucker et al. (2018), we use a power-law function to fit the rising light curves,

$\hat{F}_{\lambda}(t)=A\left(\frac{t-t_{0, \lambda}}{1 \text { day }}\right)^{B}$

where $\hat{F}_{\lambda}$ is normalized flux for each band, $t_{0, \lambda}$ is the zero point assumed for the reference epoch and $t$ is the time of photometry. We focus on the time $t_{0.01}$, representing the epoch when the light curve reaches $1 \%$ of the peak flux. Tucker et al. (2018) found that during the initial outburst, the rising in optical preceded that in the X-ray by $\Delta t=t_{0.01}^{B A T}-t_{0.01}^{V}=7.20 \pm 0.97$ days, and here we turn to study the time-lag during the rebrightening period from $t$ $\sim 370$ to 410 days. The power-law fits to the rebrightening evolution in $B$ band and Swift XRT 2-10kev band are plotted in the inset of Figure 12. Based on the fitting parameters and bootstrap-resampling technique of estimating errors, we get $t_{0.01}^{X R T}=58.92 \pm 0.15$ days for Swift XRT (2-10kev) and $t_{0.01}^{B}=38.12 \pm 2.70$ days for the $B$ band. Then we can determine a time lag between optical and X-ray outbursts as $\Delta t=t_{0.01}^{X R T}-t_{0.01}^{B}=20.80 \pm 2.85(\Delta t \pm 2 \sigma)$ days. This means that during the rebrightening process, the start of the rising in optical preceded that in the X-ray, which is consistent with the estimate for the first outburst from Tucker et al. (2018). The time-lag allows constraints on the radius $R(B)$ for the disk where the outburst is triggered and propagates simultaneously both inward and outward until reaching the inner region and producing the X-rays. We use the scaling relation in Bernardini et al. (2016) and the same parameter setup adopted in Tucker et al. (2018) to find the radius as $R(B)=[0.025-0.079] R_{\odot}(2 \sigma)$, which for the initial out- 

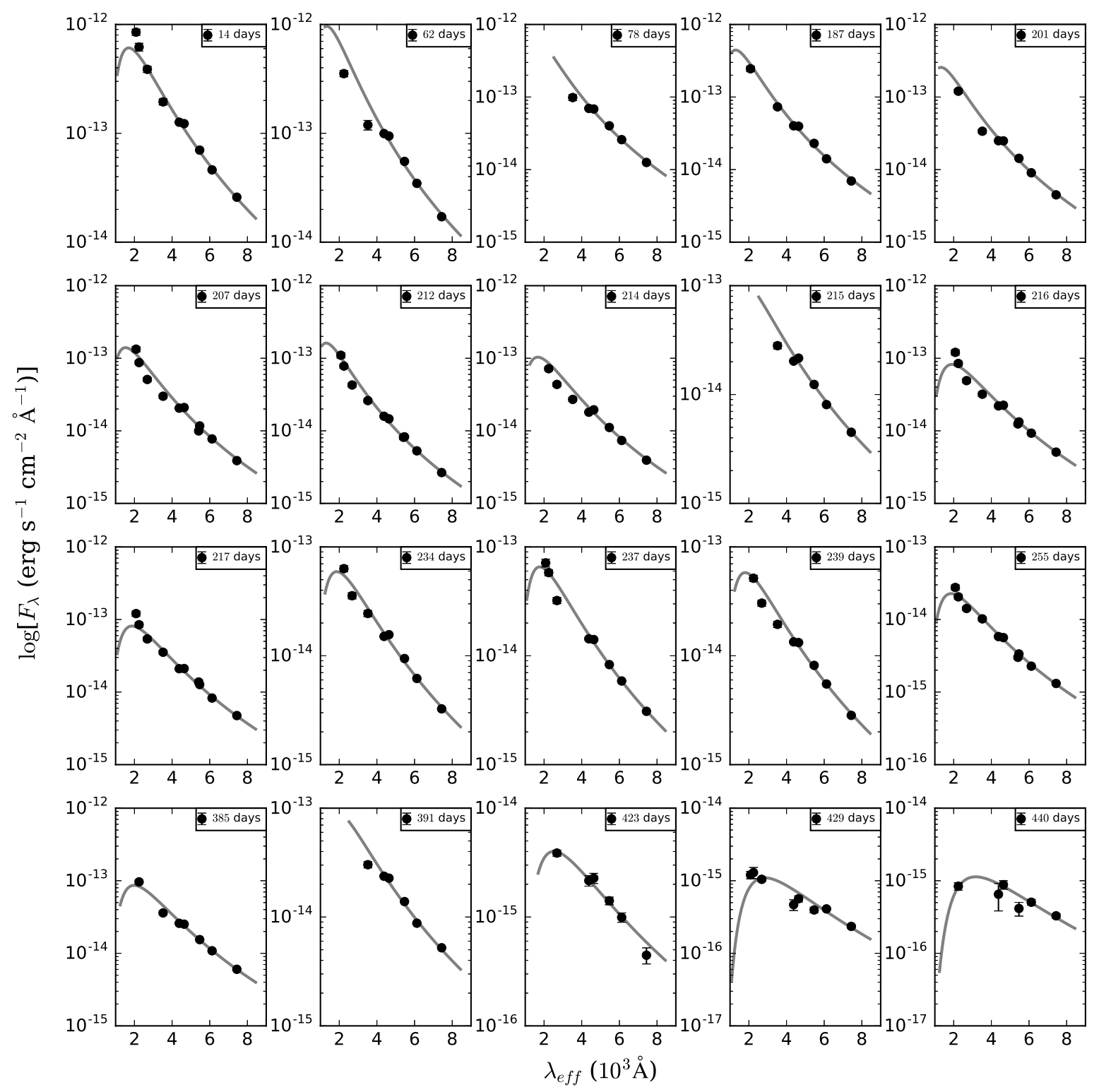

Figure 8. Fits to the SEDs of MAXI J1820+070 using a single blackbody model. Data points are presented in filled circles and the grey lines show the best-fit models.

burst is $R(V)=[0.01-0.04] R_{\odot}$, approval of the DIM and irradiation models for BH LMXB.

For MAXI J1820 +070 , one interesting evolution occurred at $t \sim 200$ to 300 days, when the emission is found to show much larger decrease in X-ray than in optical and UV bands (see also Figure 12). This observation fact suggests that the effects of irradiation on the accretion disk are diminishing during this phase, and the viscous energy of the accretion disk may contribute to maintain a lower rate of decline. Moreover, in MAXI J1820+070, an intensity jump appears in optical and UV bands at $\mathrm{t} \sim 210$ days. Augusteijn et al. (1993) reported that some LMXBs exhibited intensity jumps during the decay of the initial outburst, and they interpreted this phenomenon as an instantaneous response of the companion to the heating of X-rays and a response of the disk to the extra mass flow. The amplitude of this intensity jump tends to get stronger in redder bands (and vice versa). Note, however, this intensity jump did not occur in the X-ray band. This can be explained by a temporary increase in accretion rate of the outer disk, while this change 


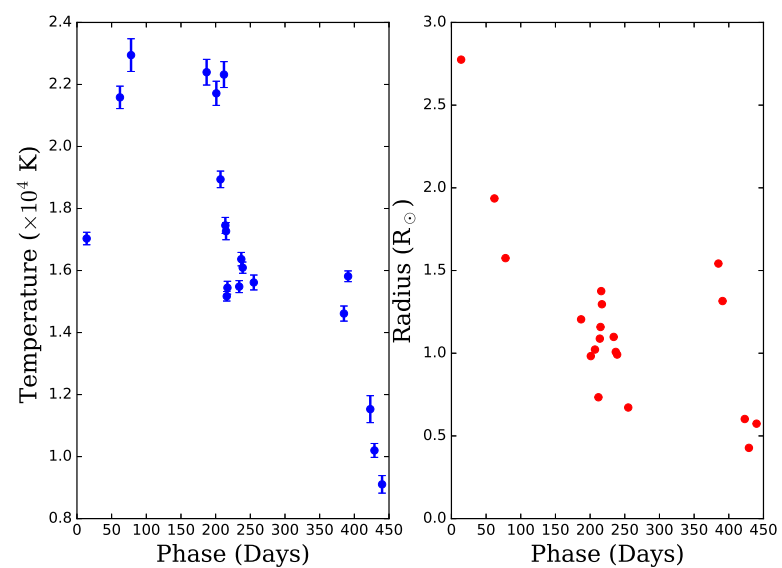

Figure 9. Evolution of temperature (left) and radius (right) inferred for MAXI J1820+070 from the blackbody fits. The blue and red dots are the best-fit values of temperature and radius, respectively.

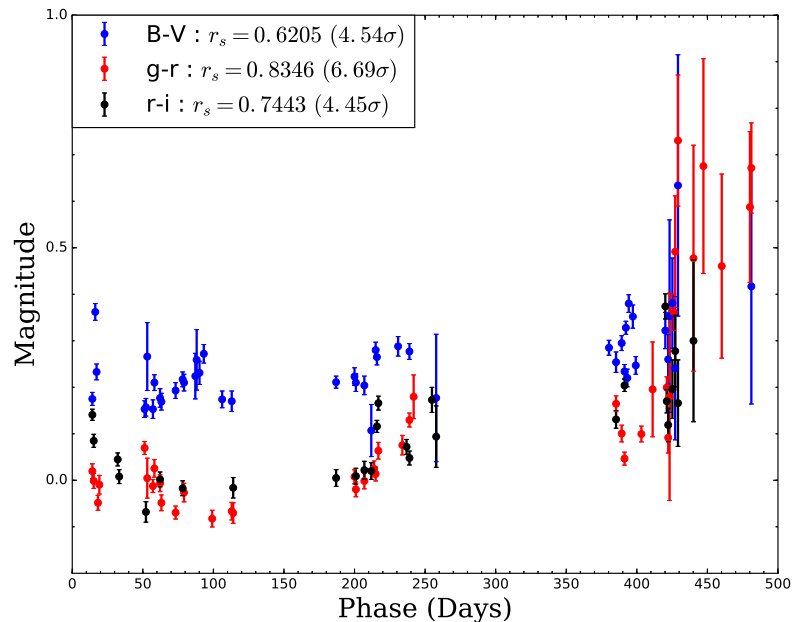

Figure 10. The $B-V, g-r$, and $r-i$ color evolution of MAXI J1820+070. $r_{s}$ in the inset window represents the coefficient of the Spearman's correlation between the color evolution and the phase, while the number in the bracket represents the significance level.

will not affect the inner disk due to a longer viscous time scale.

\section{CONCLUSIONS}

In this work, we present optical, UV and X-ray observations and studies for the BH binary system MAXI J1820+070. Several outbursts/rebrightenings were recorded during our monitoring campaign spanning from its initial optical outburst to $\sim 550$ days after. The main results from our analysis of the data are summarized as follows.

(i) The spectra of MAXI J1820+070 are characterized by

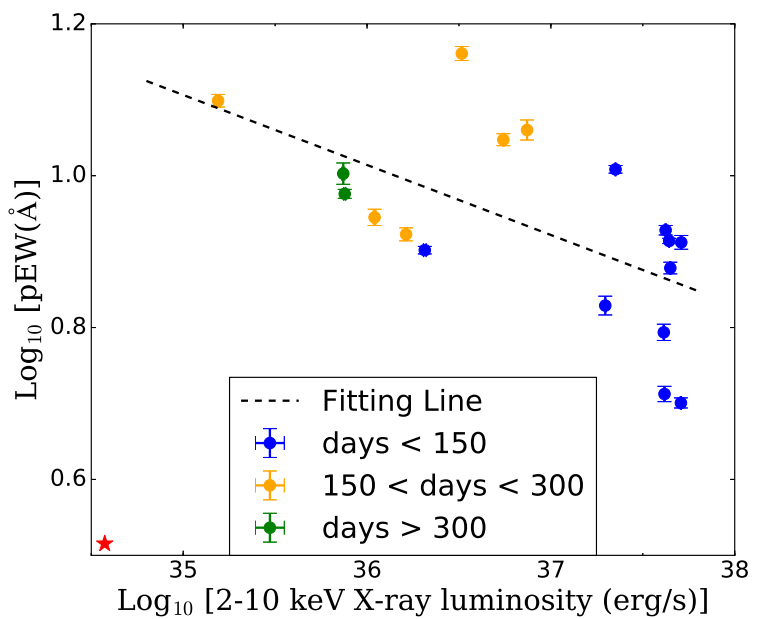

Figure 11. Relation between the $\mathrm{H}_{\alpha} \mathrm{pEW}$ measured with the spectra presented in this paper and the X-ray luminosity inferred from the Swift observations $(2-10 \mathrm{keV})$. All the data are included in our fitting except the red star at the bottom left corner. Different colors represent different phases.

blue continuum and the superimposed Balmer series, helium emission lines, and Bowen blend features, similar to those seen in several other well-known BH LMXBs like V404 Cygni (Mata Sánchez et al. 2018). The $\mathrm{H}_{\alpha}$ emission becomes progressively strong within $\sim 300$ days from the first detection of outburst, followed by a subsequent weakening during $350 \sim 400$ days. A similar trend can be also found in the $\mathrm{H}_{\beta}$ feature. The He I $\lambda 5876$ line is also found to become progressively stronger during the phase from the first detection to about 400 days after that. Such an evolution trend can be explained by the change in optical depth of continuum, as a result of the temperature change of the outer disc during the outburst.

(ii) Based on extensive optical spectroscopy and the Xray observations of MAXI J18020+070, we analyzed the relationship between the X-ray luminosity and the $\mathrm{pEW}$ of the $\mathrm{H}_{\alpha}$ emission, and we found an anticorrelation as $\mathrm{pEW}_{H_{\alpha}}=10^{4.3 \pm 1.1} L_{x}^{-0.092 \pm 0.030}$, confirming previous results.

(iii) After removing the effect of continuum, the absolute flux of $\mathrm{H}_{\alpha}, \mathrm{H}_{\beta}$ and $\mathrm{He}$ II $\lambda 4686$ showed positive correlations with the X-ray flux obtained at similar phases. This confirms that the high-energy irradiation can be regarded the pumping source for the emission of MAXI1820+070. In addition, the FWHM of $\mathrm{H}_{\beta}$ and He II $\lambda 4686$ is found to become smaller with the increase of X-ray flux but they tend to stabilize at line forming region of $1.25-1.71 R_{\odot}$, consistent with the typical radius of accretion disk around a stellar-size black hole.

(iv) The time lag found for the change in emission between optical and X-ray bands is $\Delta t=t_{0.01}^{X R T}-t_{0.01}^{B}=$ $20.80 \pm 2.85(\Delta t \pm 2 \sigma)$ days, which means that during the rebrightening process, the start of the rising in optical preceded that in the X-ray.

(v) At $\mathrm{t} \sim 200-300$ days, the X-ray flux is found to show a sudden drop, while the flux variation in optical/UV flux is 


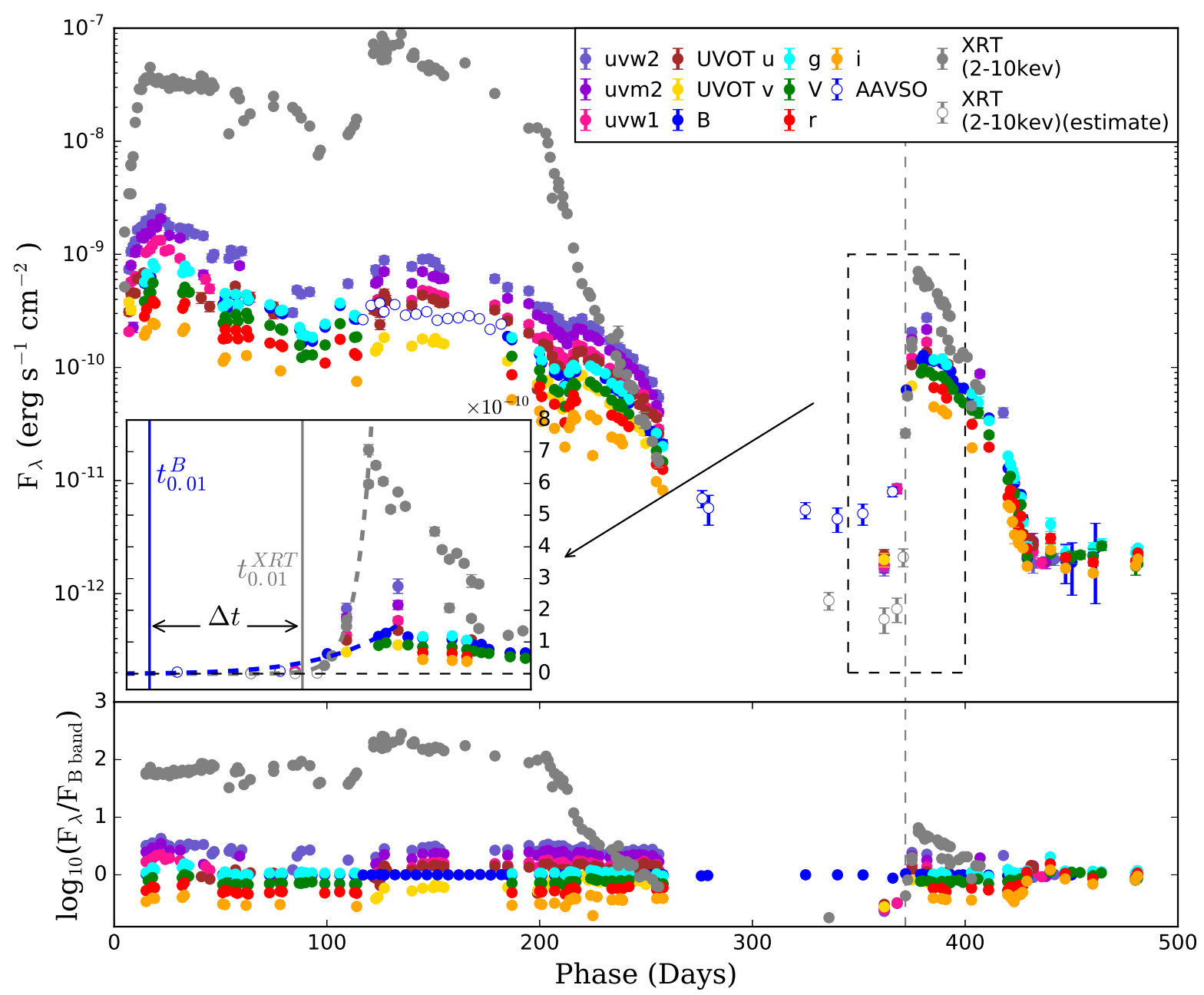

Figure 12. The upper panel shows the optical, UV and X-ray light curves of MAXI J1820+070, which are all converted into flux, while the lower panel shows the logarithmic ratio of flux in each band to the flux in B band. Different colors represent different bands, including XRT (2-10kev), uvw2, uvm2, uvw1, UVOT $u$, UVOT $v, B, g, V, r$, and $i$ bands, as shown in the legend. The phase is defined in the same way as in Figure 2. The epoch of data represented by a red star in Figure 11 is marked by grey dashed line and the specific light curve around this time (345 - 400 days) is shown in the inset. The power-law fits to the rising light curves for XRT (2-10kev) and $B$ bands are shown in dashed lines with corresponding colors. The values of $t_{0.01}$ and the time lag $(\Delta t)$ measured for the rebrightening between $B$ band and XRT (2-10kev), are also shown.

much less significant. This discrepancy suggests that the viscous energy of the accretion disk can contribute significantly to the optical/UV flux when irradiation diminished.

\section{ACKNOWLEDGEMENTS}

We thank the anonymous referee for suggestive comments to help improve this work. We acknowledge the support of the staff of the Lijiang $2.4 \mathrm{~m}$ and Xinglong $2.16 \mathrm{~m}$ telescopes. Funding for the LJT has been provided by Chinese Academy of Sciences and the People's Government of Yunnan Province. The LJT is jointly operated and administrated by Yunnan Observatories and the Center for As- tronomical Mega-Science, CAS. We acknowledge the support of the staff of the Xinglong $80 \mathrm{~cm}$ telescope. It was partially supported by the Open Project Program of the Key Laboratory of Optical Astronomy, National Astronomical Observatories, Chinese Academy of Sciences. This work is supported by the National Natural Science Foundation of China (NSFC grants 12033003, 11761141001 and 11633002), the National Program on Key Research and Development Project (grant no. 2016YFA0400803). X.W. is also supported by the Scholar Program of Beijing Academy of Science and Technology (BS2020002). J.W. acknowledges the support of the National Natural Science Foundation of China (grant No. U1938105) and the President Fund of Xi- 

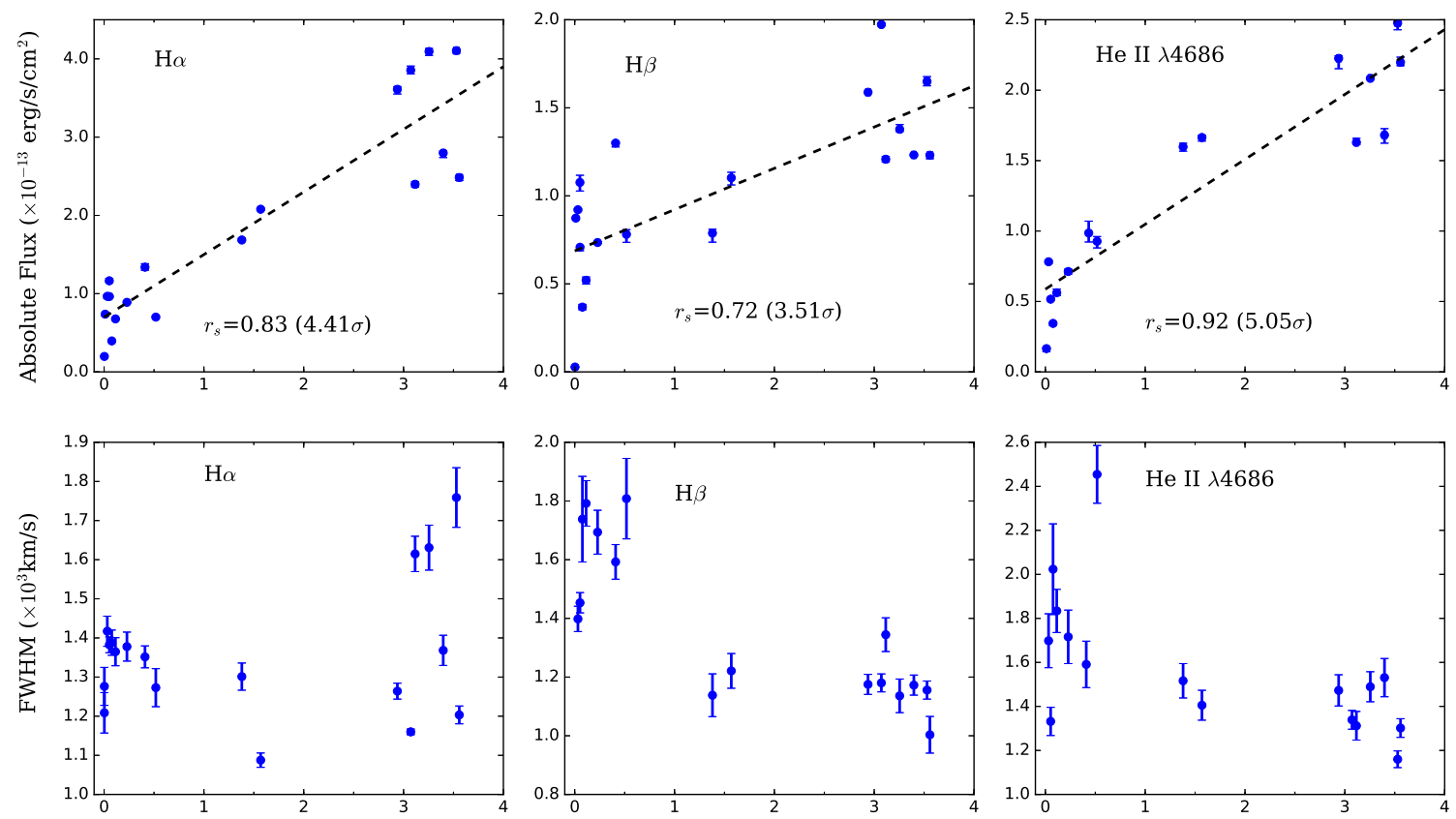

2-10 keV X-ray Flux $\left(\times 10^{-8} \mathrm{erg} / \mathrm{s} / \mathrm{cm}^{2}\right)$

Figure 13. Top panel: The relation between $2-10 \mathrm{keV}$ X-ray flux and the absolute flux of $\mathrm{H}_{\alpha}, \mathrm{H}_{\beta}$ and He II $\lambda 4686$. The black dashed lines in above three panels represent the best linear fit to the data. The results of Spearman's rank correlation test was labeled in each panel. Bottom panel: The relation between 2-10 keV X-ray flux and the FWHM of $\mathrm{H}_{\alpha}, \mathrm{H}_{\beta}$ and He iI $\lambda 4686$ lines.

amen University (No. 20720190051). Lingzhi Wang is sponsored (in part) by the Chinese Academy of Sciences (CAS), through a grant to the CAS South America Center for Astronomy (CASSACA) in Santiago, Chile. CYW is supported by the National Natural Science Foundation of China (NSFC grants 12003013). This research has made use of data provided by the Yaoan High Precision Telescope. We thank the staff of AZT for their observations and allowance of the use of the data. We acknowledge with thanks the variable star observations from the AAVSO International Database contributed by observers worldwide and used in this research.

software: ZrutyPhot (Mo et al. in prep.), IRAF (Tody 1986, 1993), SExtractor (Bertin \& Arnouts 1996)

\section{DATA AVAILABILITY}

The data underlying this article are available in the article. Our photometric data has been attached in Table A1, A2 and $\mathrm{A} 3$ in the appendix. The log table of spectral data is also shown in Table A4.

\section{REFERENCES}

Atri P., et al., 2020, MNRAS, 493, L81

Augusteijn T., Kuulkers E., Shaham J., 1993, A\&A, 279, L13

Baglio M. C., Russell D. M., Lewis F., 2018, The Astronomer's Telegram, 11418, 1
Bahramian A., Motta S., Atri P., Miller-Jones J., 2019, The Astronomer's Telegram, 12573

Bernardini F., Russell D. M., Shaw A. W., Lewis F., Charles P. A., Koljonen K. I. I., Lasota J. P., Casares J., 2016, ApJ, 818, L5 Bertin E., Arnouts S., 1996, A\&AS, 117, 393

Bright J., Motta S., Williams D., Fender R., Woudt P., MillerJones J., 2019, The Astronomer's Telegram, 13041, 1

Casares J., Jonker P. G., 2014, Space Sci. Rev., 183, 223

Casares J., Muñoz-Darias T., Mata Sánchez D., Charles P. A., Torres M. A. P., Armas Padilla M., Fender R. P., GarcíaRojas J., 2019, MNRAS, 488, 1356

Charles P. A., Coe M. J., 2006, Optical, ultraviolet and infrared observations of X-ray binaries. pp 215-265

Chen W., Shrader C. R., Livio M., 1997, ApJ, 491, 312

Corbel S., Fender R. P., 2002, ApJ, 573, L35

Dubus G., Hameury J. M., Lasota J. P., 2001, A\&A, 373, 251

Ehgamberdiev S., 2018, Nature Astronomy, 2, 349

Eikenberry S. S., Matthews K., Murphy Jr. T. W., Nelson R. W., Morgan E. H., Remillard R. A., Muno M., 1998, ApJ, 506, L31

Fender R. P., Russell D. M., Knigge C., Soria R., Hynes R. I., Goad M., 2009, MNRAS, 393, 1608

Fukugita M., Ichikawa T., Gunn J. E., Doi M., Shimasaku K., Schneider D. P., 1996, AJ, 111, 1748

Gandhi P., Rao A., Johnson M. A. C., Paice J. A., Maccarone T. J., 2019, MNRAS, 485, 2642

Gehrels N., et al., 2004, ApJ, 611, 1005

Gierliński M., Done C., Page K., 2008, MNRAS, 388, 753

Gierliński M., Done C., Page K., 2009, MNRAS, 392, 1106

Gu W.-M., et al., 2019, ApJ, 872, L20

Hambsch J., Ulowetz J., Vanmunster T., Cejudo D., Patterson J., 2019, The Astronomer's Telegram, 13014, 1 
Hankins M., et al., 2019, The Astronomer's Telegram, 13044, 1

Homan J., et al., 2018, The Astronomer's Telegram, 11820, 1

Huang F., Li J.-Z., Wang X.-F., Shang R.-C., Zhang T.-M., Hu J.-Y., Qiu Y.-L., Jiang X.-J., 2012, Research in Astronomy and Astrophysics, 12, 1585

Johnson H. L., Mitchell R. I., Iriarte B., Wisniewski W. Z., 1966, Communications of the Lunar and Planetary Laboratory, 4, 99

Kawamuro T., et al., 2018, The Astronomer's Telegram, 11399, 1

Kirsch M. G., et al., 2005, Crab: the standard x-ray candle with all (modern) x-ray satellites. pp 22-33, doi:10.1117/12.616893

Kochanek C. S., et al., 2017, PASP, 129, 104502

Lasota J.-P., 2001, New Astron. Rev., 45, 449

Leloudas G., et al., 2019, ApJ, 887, 218

Lin J., Yan Z., Han Z., Yu W., 2019, ApJ, 870, 126

Markoff S., Nowak M. A., Wilms J., 2005, ApJ, 635, 1203

Mata Sánchez D., et al., 2018, MNRAS, 481, 2646

Matsuoka M., et al., 2009, PASJ, 61, 999

Meyer F., 1986, MNRAS, 218, 7P

Mihara T., et al., 2011, PASJ, 63, S623

Muñoz-Darias T., et al., 2019, ApJ, 879, L4

Murray N., Chiang J., 1996, Nature, 382, 789

Netzer H., 2013, The Physics and Evolution of Active Galactic Nuclei. Cambridge University Press, doi:10.1017/CBO9781139109291

Osaki Y., 1995, PASJ, 47, 47

Remillard R. A., McClintock J. E., 2006, ARA\&A, 44, 49

Roming P. W. A., et al., 2005, Space Sci. Rev., 120, 95

Russell D. M., Fender R. P., Hynes R. I., Brocksopp C., Homan J., Jonker P. G., Buxton M. M., 2006, MNRAS, 371, 1334

Russell D. M., et al., 2019a, Astronomische Nachrichten, 340, 278

Russell D. M., Baglio M. C., Lewis F., 2019b, The Astronomer's Telegram, 12534

Rykoff E. S., Miller J. M., Steeghs D., Torres M. A. P., 2007, ApJ, 666,1129

Schwarz G., 1978, The Annals of Statistics, 6, 461

Shahbaz T., Bandyopadhyay R. M., Charles P. A., Wagner R. M., Muhli P., Hakala P., Casares J., Greenhill J., 1998, MNRAS, 300, 1035

Shappee B. J., et al., 2014, ApJ, 788, 48

Shidatsu M., Nakahira S., Murata K. L., Adachi R., Kawai N., Ueda Y., Negoro H., 2019, ApJ, 874, 183

Tody D., 1986, The IRAF Data Reduction and Analysis System. p. 733 , doi: $10.1117 / 12.968154$

Tody D., 1993, IRAF in the Nineties. p. 173

Tomsick J. A., Homan J., 2019, The Astronomer's Telegram, 12732

Torres M. A. P., Casares J., Jiménez-Ibarra F., Muñoz-Darias T., Armas Padilla M., Jonker P. G., Heida M., 2019, ApJ, 882, L21

Tucker M. A., et al., 2018, ApJ, 867, L9

Ulowetz J., Myers G., Patterson J., 2019, The Astronomer's Telegram, 12567

Veledina A., et al., 2019, A\&A, 623, A75

Vozza D., Ali S., Balakrishnan M., Chen J., Kebebe N., Miller J. M., Reynolds M., Tetarenko B. E., 2019, The Astronomer's Telegram, 12688

Williams R. E., 1980, ApJ, 235, 939

Wu K., Soria R., Hunstead R. W., Johnston H. M., 2001, MNRAS, 320, 177

Wu K., Soria R., Johnston H., Hunstead R., 2002, in Gurzadyan V. G., Jantzen R. T., Ruffini R., eds, The Ninth Marcel Grossmann Meeting. pp 2274-2275 (arXiv:astro-ph/0101390), doi:10.1142/9789812777386_0550

Wu J., et al., 2015, ApJ, 806, $9 \overline{2}$

Wu J., Orosz J. A., McClintock J. E., Hasan I., Bailyn C. D., Gou L., Chen Z., 2016, ApJ, 825, 46
Xu Y., Harrison F., Tomsick J., 2019, The Astronomer's Telegram, 13025, 1

Zhang J.-C., Fan Z., Yan J.-Z., Bharat Kumar Y., Li H.-B., Gao D.-Y., Jiang X.-J., 2016, PASP, 128, 105004

Zhao X., et al., 2016, ApJ, 826, 211

van Paradijs J., McClintock J. E., 1994, A\&A, 290, 133

\section{APPENDIX A: PHOTOMETRIC AND SPECTROSCOPIC DATA}

This paper has been typeset from a $\mathrm{TE}_{\mathrm{E}} \mathrm{X} / \mathrm{AT} \mathrm{EX}$ file prepared by the author. 


\section{Hanna Sai et al.}

Table A1. Photometric Standards in the MAXI J1820+070 Field

\begin{tabular}{cccccccc}
\hline Num. & $\alpha(\mathrm{J} 2000)$ & $\delta(\mathrm{J} 2000)$ & $B(\mathrm{mag})$ & $V(\mathrm{mag})$ & $g(\mathrm{mag})$ & $r(\mathrm{mag})$ & $i(\mathrm{mag})$ \\
\hline 1 & $18^{\mathrm{h}} 20^{\mathrm{m}} 25^{\mathrm{s}} .4256$ & $7^{\circ} 09^{\prime} 21^{\prime \prime} .6576$ & $14.524(068)$ & $13.828(032)$ & $14.081(002)$ & $13.647(001)$ & $13.437(001)$ \\
2 & $18^{\mathrm{h}} 20^{\mathrm{m}} 32^{\mathrm{s}} .2418$ & $7^{\circ} 15^{\prime} 02^{\prime \prime} .0196$ & $14.534(091)$ & $13.862(037)$ & $14.111(006)$ & $13.701(005)$ & $13.542(003)$ \\
3 & $18^{\mathrm{h}} 20^{\mathrm{m}} 40^{\mathrm{s}} .1472$ & $7^{\circ} 10^{\prime} 57^{\prime \prime} .8100$ & $15.361(090)$ & $13.808(033)$ & $14.419(001)$ & $13.667(030)$ & $12.982(\ldots)$ \\
4 & $18^{\mathrm{h}} 20^{\mathrm{m}} 26^{\mathrm{s}} .4202$ & $7^{\circ} 10^{\prime} 11^{\prime \prime} .7948$ & $13.976(095)$ & $12.796(046)$ & $\ldots$ & $\ldots$ & $\ldots$ \\
5 & $18^{\mathrm{h}} 20^{\mathrm{m}} 27^{\mathrm{s}} .5782$ & $7^{\circ} 11^{\prime} 40^{\prime \prime} .8696$ & $14.697(101)$ & $13.622(039)$ & $\ldots$ & $\ldots$ & $\ldots$ \\
6 & $18^{\mathrm{h}} 20^{\mathrm{m}} 25^{\mathrm{s}} .6668$ & $7^{\circ} 15^{\prime} 51^{\prime \prime} .2424$ & $14.037(101)$ & $12.805(044)$ & $\ldots$ & $\ldots$ & $\ldots$ \\
7 & $18^{\mathrm{h}} 20^{\mathrm{m}} 40^{\mathrm{s}} .0294$ & $7^{\circ} 12^{\prime} 01^{\prime \prime} .9044$ & $14.778(091)$ & $13.322(036)$ & $\ldots$ & $\ldots$ & $\ldots$ \\
8 & $18^{\mathrm{h}} 20^{\mathrm{m}} 31^{\mathrm{s}} .2221$ & $7^{\circ} 06^{\prime} 48^{\prime \prime} .8412$ & $13.792(077)$ & $12.285(030)$ & $\ldots$ & $\ldots$ & $\ldots$ \\
9 & $18^{\mathrm{h}} 20^{\mathrm{m}} 07^{\mathrm{s}} .1098$ & $7^{\circ} 13^{\prime} 28^{\prime \prime} .6068$ & $14.909(096)$ & $14.286(045)$ & $\ldots$ & $\ldots$ & $\ldots$ \\
10 & $18^{\mathrm{h}} 20^{\mathrm{m}} 10^{\mathrm{s}} .1870$ & $7^{\circ} 13^{\prime} 50^{\prime \prime} .7982$ & $\ldots$ & $\ldots$ & $14.759(002)$ & $14.189(002)$ & $13.960(001)$ \\
11 & $18^{\mathrm{h}} 20^{\mathrm{m}} 32^{\mathrm{s}} .1594$ & $7^{\circ} 07^{\prime} 04^{\prime \prime} .6632$ & $\ldots$ & $\ldots$ & $14.760(002)$ & $14.201(001)$ & $13.975(000)$ \\
12 & $18^{\mathrm{h}} 20^{\mathrm{m}} 18^{\mathrm{s}} .5156$ & $7^{\circ} 10^{\prime} 32^{\prime \prime} .7939$ & $\ldots$ & $\ldots$ & $14.851(003)$ & $14.051(001)$ & $13.687(005)$ \\
\hline
\end{tabular}

Note: Uncertainties, in units of $0.001 \mathrm{mag}$, are $1 \sigma$. 
Table A2. Ground-based Optical Photometry of MAXI J1820+070

\begin{tabular}{|c|c|c|c|c|c|c|c|c|}
\hline MJD & Phase $^{a}$ & $B$ (mag) & $g$ (mag) & $V$ (mag) & $r$ (mag) & $R$ (mag) & $i$ (mag) & data source \\
\hline 58198.3 & 14.2 & $12.573(005)$ & $12.390(010)$ & $12.398(009)$ & $12.371(006)$ & $\ldots$ & $12.230(006)$ & TNT \\
\hline 58199.3 & 15.2 & $12.430(008)$ & $12.212(008)$ & $\ldots$ & $12.214(008)$ & $\ldots$ & $12.129(006)$ & TNT \\
\hline 58200.4 & 16.3 & $12.558(012)$ & $12.294(014)$ & $12.196(006)$ & $\ldots$ & $\ldots$ & $\ldots$ & TNT \\
\hline 58201.3 & 17.2 & $12.433(007)$ & $\ldots$ & $12.200(010)$ & $\ldots$ & $\ldots$ & $\ldots$ & TNT \\
\hline 58202.3 & 18.2 & $\ldots$ & $11.983(007)$ & $\ldots$ & $12.032(009)$ & $\ldots$ & $11.965(010)$ & TNT \\
\hline 58202.4 & 18.3 & $\ldots$ & $\ldots$ & $11.987(007)$ & $\ldots$ & $\ldots$ & $\ldots$ & TNT \\
\hline 58203.3 & 19.2 & $\ldots$ & $12.074(010)$ & $\ldots$ & $12.084(010)$ & $\ldots$ & 11.991(009) & TNT \\
\hline 58216.3 & 32.2 & $\ldots$ & $12.185(006)$ & $12.206(007)$ & $12.191(009)$ & $\ldots$ & $12.146(005)$ & TNT \\
\hline 58217.3 & 33.2 & $\ldots$ & $12.034(006)$ & $12.081(005)$ & $12.080(006)$ & $\ldots$ & $12.072(009)$ & TNT \\
\hline 58219.4 & 35.3 & $\ldots$ & $12.153(007)$ & $12.193(008)$ & $\ldots$ & $\ldots$ & $\ldots$ & TNT \\
\hline 58235.3 & 51.2 & $13.034(008)$ & $12.936(007)$ & $12.881(010)$ & $12.867(007)$ & $\ldots$ & $12.805(008)$ & TNT \\
\hline 58236.3 & 52.2 & $12.892(011)$ & $12.641(011)$ & $12.735(008)$ & $12.660(011)$ & $\ldots$ & $12.728(011)$ & TNT \\
\hline 58237.2 & 53.1 & $13.118(040)$ & $12.858(030)$ & $12.852(033)$ & $12.854(013)$ & $\ldots$ & $\ldots$ & TNT \\
\hline 58241.2 & 57.1 & $12.836(012)$ & $12.662(007)$ & $12.683(008)$ & $12.675(007)$ & $\ldots$ & $\ldots$ & TNT \\
\hline 58242.3 & 58.2 & $13.042(009)$ & $12.891(008)$ & $12.832(008)$ & $12.866(011)$ & $\ldots$ & $\ldots$ & TNT \\
\hline 58246.3 & 62.2 & $12.834(011)$ & 12.674(009) & $12.657(009)$ & $12.681(009)$ & $\ldots$ & $12.679(007)$ & TNT \\
\hline 58247.2 & 63.1 & $12.945(011)$ & $12.777(010)$ & $12.776(007)$ & $12.826(007)$ & $\ldots$ & $\ldots$ & TNT \\
\hline 58257.3 & 73.2 & $13.119(010)$ & $12.890(007)$ & $12.926(007)$ & $12.960(007)$ & $\ldots$ & $\ldots$ & TNT \\
\hline 58262.3 & 78.2 & $13.220(009)$ & $13.025(008)$ & $13.003(007)$ & $13.000(009)$ & $\ldots$ & $13.017(007)$ & TNT \\
\hline 58263.3 & 79.2 & $13.244(010)$ & $13.012(011)$ & $13.034(008)$ & $13.039(009)$ & $\ldots$ & $\ldots$ & TNT \\
\hline 58271.3 & 87.2 & $13.591(028)$ & $13.394(019)$ & $13.367(021)$ & $\ldots$ & $\ldots$ & $\ldots$ & TNT \\
\hline 58272.2 & 88.1 & $13.894(036)$ & $13.565(025)$ & $13.635(029)$ & $\ldots$ & $\ldots$ & $\ldots$ & TNT \\
\hline 58274.2 & 90.1 & $13.811(013)$ & $13.578(008)$ & $13.580(012)$ & $\ldots$ & $\ldots$ & $\ldots$ & TNT \\
\hline 58277.3 & 93.2 & $13.852(011)$ & $13.612(010)$ & $13.580(009)$ & $\ldots$ & $\ldots$ & $\ldots$ & TNT \\
\hline 58283.2 & 99.1 & $13.540(008)$ & $13.319(009)$ & $13.360(007)$ & $13.402(009)$ & $\ldots$ & $\ldots$ & TNT \\
\hline 58290.3 & 106.2 & $13.063(007)$ & $12.855(007)$ & $12.889(011)$ & $12.878(010)$ & $\ldots$ & $\ldots$ & TNT \\
\hline 58297.1 & 113.0 & $13.362(012)$ & $13.126(009)$ & $13.192(010)$ & $13.193(010)$ & $\ldots$ & $\ldots$ & TNT \\
\hline 58298.1 & 114.0 & $13.366(013)$ & $13.163(010)$ & $13.181(011)$ & $13.234(012)$ & $\ldots$ & $13.250(010)$ & TNT \\
\hline 58300.5 & 116.4 & $13.374(010)$ & $\ldots$ & $\ldots$ & $\ldots$ & $\ldots$ & $\ldots$ & AAVSO \\
\hline 58304.3 & 120.2 & $13.061(011)$ & $\ldots$ & $\ldots$ & $\ldots$ & $\ldots$ & $\ldots$ & AAVSO \\
\hline 58308.4 & 124.3 & $13.010(065)$ & $\ldots$ & $\ldots$ & $\ldots$ & $\ldots$ & $\ldots$ & AAVSO \\
\hline 58310.4 & 126.3 & $13.197(045)$ & $\ldots$ & $\ldots$ & $\ldots$ & $\ldots$ & $\ldots$ & AAVSO \\
\hline 58315.5 & 131.4 & $13.042(007)$ & $\ldots$ & $\ldots$ & $\ldots$ & $\ldots$ & $\ldots$ & AAVSO \\
\hline 58320.5 & 136.4 & $13.276(009)$ & $\ldots$ & $\ldots$ & $\ldots$ & $\ldots$ & $\ldots$ & AAVSO \\
\hline 58325.5 & 141.4 & $13.253(009)$ & $\ldots$ & $\ldots$ & $\ldots$ & $\ldots$ & $\ldots$ & AAVSO \\
\hline 58330.5 & 146.4 & $13.200(014)$ & $\ldots$ & $\ldots$ & $\ldots$ & $\ldots$ & $\ldots$ & AAVSO \\
\hline 58335.4 & 151.3 & $13.388(028)$ & $\ldots$ & $\ldots$ & $\ldots$ & $\ldots$ & $\ldots$ & AAVSO \\
\hline 58340.4 & 156.3 & $13.352(026)$ & $\ldots$ & $\ldots$ & $\ldots$ & $\ldots$ & $\ldots$ & AAVSO \\
\hline 58345.3 & 161.2 & $13.335(013)$ & $\ldots$ & $\ldots$ & $\ldots$ & $\ldots$ & $\ldots$ & AAVSO \\
\hline 58350.5 & 166.4 & $13.289(010)$ & $\ldots$ & $\ldots$ & $\ldots$ & $\ldots$ & $\ldots$ & AAVSO \\
\hline 58355.3 & 171.2 & $13.355(017)$ & $\ldots$ & $\ldots$ & $\ldots$ & $\ldots$ & $\ldots$ & AAVSO \\
\hline 58360.3 & 176.2 & $13.588(014)$ & $\ldots$ & $\ldots$ & $\ldots$ & $\ldots$ & $\ldots$ & AAVSO \\
\hline 58365.5 & 181.4 & $13.473(012)$ & $\ldots$ & $\ldots$ & $\ldots$ & $\ldots$ & $\ldots$ & AAVSO \\
\hline 58369.0 & 184.9 & $13.745(008)$ & $\ldots$ & $\ldots$ & $\ldots$ & $\ldots$ & $\ldots$ & TNT \\
\hline 58371.0 & 186.9 & $13.820(006)$ & $13.618(007)$ & $13.609(007)$ & $13.660(010)$ & $\ldots$ & $13.655(008)$ & TNT \\
\hline 58384.0 & 199.9 & $14.142(008)$ & $13.928(007)$ & $13.919(011)$ & $13.921(008)$ & $\ldots$ & $\ldots$ & TNT \\
\hline 58384.1 & 200.0 & $\ldots$ & $\ldots$ & $\ldots$ & $\ldots$ & $\ldots$ & $13.907(010)$ & TNT \\
\hline 58385.0 & 200.9 & $14.334(008)$ & $14.122(007)$ & $14.124(011)$ & $14.142(009)$ & $\ldots$ & $14.133(008)$ & TNT \\
\hline 58391.0 & 206.9 & $14.547(010)$ & $14.309(008)$ & $14.343(010)$ & $14.311(009)$ & $\ldots$ & $14.289(010)$ & TNT \\
\hline 58393.0 & 208.9 & $14.602(010)$ & $\ldots$ & $14.400(006)$ & $\ldots$ & $\ldots$ & $\ldots$ & TNT \\
\hline 58395.9 & 211.8 & $14.826(037)$ & $14.698(013)$ & $14.719(019)$ & $\ldots$ & $\ldots$ & $\ldots$ & TNT \\
\hline 58396.0 & 211.9 & $\ldots$ & $\ldots$ & $\ldots$ & $14.721(011)$ & $\ldots$ & $14.701(007)$ & TNT \\
\hline 58397.9 & 213.8 & $14.687(030)$ & $14.387(008)$ & $14.397(010)$ & $14.363(009)$ & $\ldots$ & $\ldots$ & TNT \\
\hline 58398.0 & 213.9 & $\ldots$ & $\ldots$ & $\ldots$ & $\ldots$ & $\ldots$ & $14.274(010)$ & TNT \\
\hline 58399.0 & 214.9 & $14.560(010)$ & $14.276(006)$ & $14.280(007)$ & $14.263(009)$ & $\ldots$ & $\ldots$ & TNT \\
\hline 58399.1 & 215.0 & $\ldots$ & $\ldots$ & $\ldots$ & $\ldots$ & $\ldots$ & $14.129(010)$ & TNT \\
\hline 58400.0 & 215.9 & $14.470(010)$ & $14.230(007)$ & $14.205(007)$ & $14.112(007)$ & $\ldots$ & $13.996(006)$ & TNT \\
\hline 58401.0 & 216.9 & $14.523(010)$ & $14.301(010)$ & $14.253(010)$ & $14.238(008)$ & $\ldots$ & $14.072(007)$ & TNT \\
\hline 58408.0 & 223.9 & $\ldots$ & $14.246(007)$ & $14.179(007)$ & $\ldots$ & $\ldots$ & $14.000(007)$ & TNT \\
\hline 58409.0 & 224.9 & $\ldots$ & $\ldots$ & $\ldots$ & $\ldots$ & $\ldots$ & $14.890(054)$ & TNT \\
\hline 58410.0 & 225.9 & $\ldots$ & $14.302(008)$ & $14.254(009)$ & $\ldots$ & $\ldots$ & $\ldots$ & TNT \\
\hline 58411.0 & 226.9 & $\ldots$ & $14.340(008)$ & $14.291(008)$ & $\ldots$ & $\ldots$ & $14.099(008)$ & TNT \\
\hline
\end{tabular}

a Days relative to the initial optical outburst (UT March 06.58 $2018=$ MJD 58184.08).

Note: Uncertainties, in units of $0.001 \mathrm{mag}$, are $1 \sigma$. 
Table A2 - continued A table continued from the previous one.

\begin{tabular}{|c|c|c|c|c|c|c|c|c|}
\hline MJD & Phase $^{a}$ & $B$ (mag) & $g$ (mag) & $V$ (mag) & $r$ (mag) & $R$ (mag) & $i$ (mag) & data source \\
\hline 58415.0 & 230.9 & $14.674(012)$ & $14.413(008)$ & $14.386(009)$ & $\ldots$ & $\ldots$ & $\ldots$ & TNT \\
\hline 58418.0 & 233.9 & $14.883(010)$ & $14.627(011)$ & $14.575(011)$ & $14.552(010)$ & $\ldots$ & $14.482(010)$ & TNT \\
\hline 58420.9 & 236.8 & $14.938(026)$ & $14.739(011)$ & $14.715(011)$ & $14.610(009)$ & $\ldots$ & $14.538(007)$ & TNT \\
\hline 58422.0 & 237.9 & $\ldots$ & $14.685(009)$ & $14.655(009)$ & $\ldots$ & $\ldots$ & $14.526(011)$ & TNT \\
\hline 58423.0 & 238.9 & $15.009(009)$ & $14.808(007)$ & $14.732(008)$ & $14.679(008)$ & $\ldots$ & $14.631(007)$ & TNT \\
\hline 58426.0 & 241.9 & $15.223(030)$ & $14.985(027)$ & $14.887(025)$ & $14.806(020)$ & $\ldots$ & $\ldots$ & TNT \\
\hline 58436.0 & 251.9 & $15.833(095)$ & $\ldots$ & $\ldots$ & $\ldots$ & $\ldots$ & $\ldots$ & TNT \\
\hline 58438.9 & 254.8 & $15.916(029)$ & $15.735(014)$ & $15.699(015)$ & $15.639(013)$ & $\ldots$ & $15.466(014)$ & TNT \\
\hline 58441.9 & 257.8 & $16.119(090)$ & $16.024(056)$ & $15.942(047)$ & $15.752(034)$ & $\ldots$ & $15.658(032)$ & TNT \\
\hline 58523.5 & 339.4 & $17.776(264)$ & $\ldots$ & $\ldots$ & $\ldots$ & $\ldots$ & $\ldots$ & AAVSO \\
\hline 58535.4 & 351.3 & $17.661(230)$ & $\ldots$ & $\ldots$ & $\ldots$ & $\ldots$ & $\ldots$ & AAVSO \\
\hline 58549.4 & 365.3 & $17.177(108)$ & $\ldots$ & $\ldots$ & $\ldots$ & $\ldots$ & $\ldots$ & AAVSO \\
\hline 58556.4 & 372.3 & $14.930(009)$ & $\ldots$ & $\ldots$ & $\ldots$ & $\ldots$ & $\ldots$ & TNT \\
\hline 58563.4 & 379.3 & $14.254(011)$ & $\ldots$ & $13.980(009)$ & $\ldots$ & $\ldots$ & $\ldots$ & TNT \\
\hline 58564.4 & 380.3 & $14.167(009)$ & $\ldots$ & $13.882(007)$ & $\ldots$ & $\ldots$ & $\ldots$ & TNT \\
\hline 58567.4 & 383.3 & $14.269(007)$ & $\ldots$ & $13.960(007)$ & $\ldots$ & $\ldots$ & $\ldots$ & TNT \\
\hline 58569.4 & 385.3 & $14.294(012)$ & $14.108(009)$ & $14.040(010)$ & $13.944(008)$ & $\ldots$ & $13.813(011)$ & TNT \\
\hline 58573.4 & 389.3 & $14.345(007)$ & $14.085(009)$ & $14.050(009)$ & $13.985(009)$ & $\ldots$ & $13.888(009)$ & TNT \\
\hline 58575.4 & 391.3 & $14.391(007)$ & $14.219(008)$ & $14.157(008)$ & $14.173(006)$ & $\ldots$ & $13.969(007)$ & TNT \\
\hline 58576.3 & 392.2 & $14.548(006)$ & $\ldots$ & $14.220(008)$ & $\ldots$ & $\ldots$ & $\ldots$ & TNT \\
\hline 58577.4 & 393.3 & $14.516(010)$ & $\ldots$ & $14.296(009)$ & $\ldots$ & $\ldots$ & $\ldots$ & TNT \\
\hline 58578.4 & 394.3 & $14.720(011)$ & $\ldots$ & $14.340(008)$ & $\ldots$ & $\ldots$ & $\ldots$ & TNT \\
\hline 58581.3 & 397.2 & $14.889(012)$ & $\ldots$ & $14.537(013)$ & $\ldots$ & $\ldots$ & $\ldots$ & TNT \\
\hline 58583.3 & 399.2 & $14.888(008)$ & $\ldots$ & $14.641(011)$ & $\ldots$ & $\ldots$ & $\ldots$ & TNT \\
\hline 58587.3 & 403.2 & $15.124(009)$ & $14.853(008)$ & $14.801(007)$ & $14.754(009)$ & $\ldots$ & $14.718(009)$ & TNT \\
\hline 58590.3 & 406.2 & $\ldots$ & $15.051(131)$ & $14.858(116)$ & $\ldots$ & $\ldots$ & $\ldots$ & TNT \\
\hline 58595.3 & 411.2 & $15.548(043)$ & $15.456(056)$ & $15.348(054)$ & $15.261(046)$ & $\ldots$ & $\ldots$ & TNT \\
\hline 58604.3 & 420.2 & $16.659(023)$ & $16.236(011)$ & $16.337(016)$ & $16.363(015)$ & $\ldots$ & $15.989(012)$ & TNT \\
\hline 58605.3 & 421.2 & $16.592(020)$ & $16.410(012)$ & $16.260(015)$ & $16.211(011)$ & $\ldots$ & $16.041(014)$ & TNT \\
\hline 58606.3 & 422.2 & $16.864(031)$ & $16.560(018)$ & $16.604(022)$ & $16.469(015)$ & $\ldots$ & $16.350(021)$ & TNT \\
\hline 58607.3 & 423.2 & $16.992(119)$ & $16.718(116)$ & $16.639(088)$ & $16.539(107)$ & $\ldots$ & $16.640(183)$ & TNT \\
\hline 58609.3 & 425.2 & $17.496(058)$ & $17.229(031)$ & $17.115(039)$ & $17.026(029)$ & $\ldots$ & $16.830(034)$ & TNT \\
\hline 58610.3 & 426.2 & $17.239(032)$ & $17.150(022)$ & $16.889(023)$ & $16.789(017)$ & $\ldots$ & $16.657(026)$ & TNT \\
\hline 58611.2 & 427.1 & $17.778(089)$ & $\ldots$ & $17.537(065)$ & $\ldots$ & $\ldots$ & $\ldots$ & TNT \\
\hline 58611.3 & 427.2 & $\ldots$ & $17.699(079)$ & $\ldots$ & $17.208(041)$ & $\ldots$ & $16.930(036)$ & TNT \\
\hline 58613.2 & 429.1 & $18.653(184)$ & $18.232(104)$ & $18.019(097)$ & $17.502(037)$ & $\ldots$ & $17.336(056)$ & TNT \\
\hline 58624.2 & 440.1 & $18.294(446)$ & $17.748(145)$ & $17.968(233)$ & $17.271(098)$ & $\ldots$ & $16.971(076)$ & TNT \\
\hline 58631.3 & 447.2 & $18.692(412)$ & $18.375(168)$ & $18.068(106)$ & $17.700(063)$ & $\ldots$ & $17.382(068)$ & TNT \\
\hline 58634.3 & 450.2 & $18.740(529)$ & $\ldots$ & $18.045(133)$ & $\ldots$ & $\ldots$ & $\ldots$ & TNT \\
\hline 58638.3 & 454.2 & $\ldots$ & $\ldots$ & $18.001(086)$ & $\ldots$ & $\ldots$ & $\ldots$ & TNT \\
\hline 58644.2 & 460.1 & $18.573(160)$ & $18.266(120)$ & $18.035(176)$ & $17.806(078)$ & $\ldots$ & $\ldots$ & TNT \\
\hline 58644.3 & 460.2 & $\ldots$ & $\ldots$ & $\ldots$ & $\ldots$ & $\ldots$ & $17.487(058)$ & TNT \\
\hline 58645.3 & 461.2 & $18.436(732)$ & $\ldots$ & $\ldots$ & $\ldots$ & $\ldots$ & $\ldots$ & TNT \\
\hline 58648.2 & 464.1 & $\ldots$ & $18.174(118)$ & $17.801(095)$ & $\ldots$ & $\ldots$ & $\ldots$ & TNT \\
\hline 58663.4 & 479.3 & $18.623(030)$ & $\ldots$ & $17.954(039)$ & $\ldots$ & $\ldots$ & $\ldots$ & $\mathrm{AZT}$ \\
\hline 58664.2 & 480.1 & $\ldots$ & $18.368(115)$ & $18.250(182)$ & $17.781(047)$ & $\ldots$ & $17.320(048)$ & TNT \\
\hline 58665.2 & 481.1 & $18.607(187)$ & $18.272(060)$ & $18.190(066)$ & $17.601(037)$ & $\ldots$ & $17.179(035)$ & TNT \\
\hline 58669.8 & 485.7 & $18.620(027)$ & $\ldots$ & $17.949(031)$ & $\ldots$ & $\ldots$ & $\ldots$ & $\mathrm{AZT}$ \\
\hline 58675.3 & 491.2 & $18.612(032)$ & $\ldots$ & $17.961(028)$ & $\ldots$ & $\ldots$ & $\ldots$ & $\mathrm{AZT}$ \\
\hline 58684.7 & 500.6 & $18.940(028)$ & $\ldots$ & $18.183(029)$ & $\ldots$ & $\ldots$ & $\ldots$ & $\mathrm{AZT}$ \\
\hline 58708.7 & 524.6 & $15.552(005)$ & $\ldots$ & $\ldots$ & $\ldots$ & $14.959(003)$ & $\ldots$ & Yaoan \\
\hline 58710.7 & 526.6 & $14.836(004)$ & $\ldots$ & $\ldots$ & $\ldots$ & $14.324(002)$ & $\ldots$ & Yaoan \\
\hline 58711.7 & 527.6 & $14.833(004)$ & $\ldots$ & $\ldots$ & $\ldots$ & $14.299(002)$ & $\ldots$ & Yaoan \\
\hline 58720.8 & 536.7 & $14.395(012)$ & $\ldots$ & $\ldots$ & $\ldots$ & $13.667(003)$ & $\ldots$ & Yaoan \\
\hline
\end{tabular}

Note: Uncertainties, in units of $0.001 \mathrm{mag}$, are $1 \sigma$. 
Table A3. Swift Photometry of MAXI J1820+070

\begin{tabular}{|c|c|c|c|c|c|c|}
\hline MJD & Phase $^{a}$ & uvw2 (mag) & uvm2 (mag) & uvw1 (mag) & UVOT u (mag) & UVOT v (mag) \\
\hline 58191 & 7 & $12.37(07)$ & $\ldots$ & $13.10(06)$ & $12.52(07)$ & $12.46(07)$ \\
\hline 58192 & 8 & $11.98(09)$ & $12.18(07)$ & $12.00(07)$ & $12.12(07)$ & $12.63(09)$ \\
\hline 58193 & 9 & $11.86(07)$ & $13.55(11)$ & 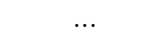 & $12.10(07)$ & $\ldots$ \\
\hline 58194 & 10 & $11.75(07)$ & $11.90(09)$ & $11.88(08)$ & $12.11(07)$ & $\ldots$ \\
\hline 58195 & 11 & $11.50(07)$ & $11.84(09)$ & $\ldots$ & $11.79(07)$ & $\ldots$ \\
\hline 58197 & 13 & $11.43(07)$ & $11.55(09)$ & $\ldots$ & $11.76(07)$ & $\ldots$ \\
\hline 58198 & 14 & $11.42(07)$ & $11.58(09)$ & $\ldots$ & $11.66(07)$ & $\ldots$ \\
\hline 58199 & 15 & $11.30(09)$ & $11.48(09)$ & $11.35(07)$ & $\ldots$ & $\ldots$ \\
\hline 58201 & 17 & $11.34(09)$ & $11.49(07)$ & $11.28(07)$ & $\ldots$ & $\ldots$ \\
\hline 58202 & 18 & $11.17(09)$ & $11.28(07)$ & $11.19(07)$ & $\ldots$ & $\ldots$ \\
\hline 58204 & 20 & $11.24(09)$ & $11.34(07)$ & $11.09(07)$ & $\ldots$ & $\ldots$ \\
\hline 58206 & 22 & $11.03(09)$ & $11.15(07)$ & $11.08(08)$ & $\ldots$ & $\ldots$ \\
\hline 58208 & 24 & $11.32(09)$ & $\ldots$ & $11.32(07)$ & $\ldots$ & $\ldots$ \\
\hline 58210 & 26 & $11.42(09)$ & $11.52(07)$ & $11.29(07)$ & $\ldots$ & $\ldots$ \\
\hline 58215 & 31 & $11.46(09)$ & $11.58(07)$ & $11.48(07)$ & $\ldots$ & $\ldots$ \\
\hline 58217 & 33 & 11.62(09) & $\ldots$ & $\ldots$ & $\ldots$ & $\ldots$ \\
\hline 58219 & 35 & $11.48(09)$ & $\ldots$ & $\ldots$ & $\ldots$ & $\ldots$ \\
\hline 58222 & 38 & $11.59(09)$ & $\ldots$ & $\ldots$ & $\ldots$ & $\ldots$ \\
\hline 58225 & 41 & $\ldots$ & $\ldots$ & $\ldots$ & $12.21(12)$ & $\ldots$ \\
\hline 58226 & 42 & $11.63(09)$ & $12.39(10)$ & $\ldots$ & $\ldots$ & $\ldots$ \\
\hline 58227 & 43 & $\ldots$ & $\ldots$ & $11.94(10)$ & $\ldots$ & $\ldots$ \\
\hline 58229 & 45 & $\ldots$ & $\ldots$ & $12.15(10)$ & $12.40(11)$ & $\ldots$ \\
\hline 58230 & 46 & $12.12(09)$ & $\ldots$ & $\ldots$ & $\ldots$ & $\ldots$ \\
\hline 58231 & 47 & $12.04(09)$ & $\ldots$ & $\ldots$ & $\ldots$ & $\ldots$ \\
\hline 58238 & 54 & $12.13(09)$ & $\ldots$ & $\ldots$ & $\ldots$ & $\ldots$ \\
\hline 58239 & 55 & $11.95(09)$ & $\ldots$ & $\ldots$ & $\ldots$ & $\ldots$ \\
\hline 58241 & 57 & $12.04(09)$ & $\ldots$ & $\ldots$ & 11.95(07) & $\ldots$ \\
\hline 58243 & 59 & $\ldots$ & $12.19(07)$ & $\ldots$ & $\ldots$ & $\ldots$ \\
\hline 58244 & 60 & $11.97(09)$ & $\ldots$ & $\ldots$ & $\ldots$ & $\ldots$ \\
\hline 58248 & 64 & $\ldots$ & $\ldots$ & $\ldots$ & $12.19(11)$ & $\ldots$ \\
\hline 58259 & 75 & $\ldots$ & $\ldots$ & $\ldots$ & $12.56(11)$ & $\ldots$ \\
\hline 58259 & 75 & $\ldots$ & $\ldots$ & $\ldots$ & $12.40(10)$ & $\ldots$ \\
\hline 58268 & 84 & 13.33(09) & $\ldots$ & $\ldots$ & $\ldots$ & $\ldots$ \\
\hline 58270 & 86 & $12.83(09)$ & $\ldots$ & $\ldots$ & $\ldots$ & $\ldots$ \\
\hline 58272 & 88 & $12.92(09)$ & $\ldots$ & $\ldots$ & $\ldots$ & $\ldots$ \\
\hline 58276 & 92 & $12.87(09)$ & $\ldots$ & $\ldots$ & ... & $\cdots$ \\
\hline 58294 & 110 & $12.69(09)$ & $\ldots$ & $\ldots$ & $\ldots$ & $\ldots$ \\
\hline 58306 & 122 & $\ldots$ & $\ldots$ & $\ldots$ & $12.46(10)$ & $\ldots$ \\
\hline 58307 & 123 & $12.38(09)$ & $12.58(07)$ & $12.49(07)$ & $12.50(07)$ & $13.54(06)$ \\
\hline 58308 & 124 & $12.50(09)$ & $12.54(07)$ & $12.39(07)$ & $12.31(07)$ & $13.46(06)$ \\
\hline 58309 & 125 & $\ldots$ & $\ldots$ & $\ldots$ & $12.67(11)$ & $\ldots$ \\
\hline 58310 & 126 & $\ldots$ & $\ldots$ & $\ldots$ & $12.45(11)$ & $\ldots$ \\
\hline 58311 & 127 & $12.17(09)$ & $12.32(07)$ & $12.28(07)$ & 12.17(09) & $13.24(06)$ \\
\hline 58312 & 128 & $\ldots$ & $\ldots$ & $\ldots$ & $12.26(11)$ & $\ldots$ \\
\hline 58324 & 140 & $\ldots$ & $12.58(07)$ & $12.41(07)$ & $12.38(08)$ & $13.43(06)$ \\
\hline 58329 & 145 & $12.15(09)$ & $12.32(07)$ & $12.19(07)$ & $12.15(08)$ & $13.27(06)$ \\
\hline 58332 & 148 & $12.14(09)$ & $\ldots$ & $12.21(07)$ & $\ldots$ & $\ldots$ \\
\hline 58334 & 150 & $\ldots$ & $12.43(07)$ & $12.25(07)$ & $12.23(08)$ & $13.34(06)$ \\
\hline 58335 & 151 & $12.22(09)$ & $12.42(07)$ & $12.30(07)$ & $12.25(08)$ & $13.26(06)$ \\
\hline 58337 & 153 & $12.37(09)$ & $12.48(07)$ & $12.30(07)$ & $12.32(08)$ & 13.35(06) \\
\hline 58339 & 155 & $\ldots$ & $12.47(07)$ & $12.33(07)$ & $12.32(07)$ & $13.38(06)$ \\
\hline 58363 & 179 & $12.58(09)$ & $\ldots$ & $12.51(07)$ & $12.48(07)$ & $\ldots$ \\
\hline 58369 & 185 & $12.77(09)$ & $12.91(07)$ & $12.80(07)$ & $12.72(07)$ & $13.76(06)$ \\
\hline 58379 & 195 & $12.85(09)$ & $13.03(07)$ & $12.94(07)$ & $12.99(07)$ & $\ldots$ \\
\hline 58383 & 199 & $13.20(09)$ & $13.28(07)$ & $13.25(07)$ & $14.23(06)$ & $14.27(06)$ \\
\hline 58386 & 202 & $13.21(09)$ & 13.36(07) & $13.22(07)$ & $13.23(07)$ & $14.26(06)$ \\
\hline 58388 & 204 & $13.22(09)$ & $13.38(07)$ & $13.26(07)$ & $13.28(07)$ & $14.19(06)$ \\
\hline 58390 & 206 & $13.39(09)$ & $13.56(07)$ & 13.53(07) & $13.50(07)$ & $14.52(06)$ \\
\hline 58391 & 207 & $13.43(09)$ & $13.71(07)$ & $13.55(07)$ & $13.69(07)$ & $14.57(06)$ \\
\hline 58393 & 209 & $13.49(09)$ & $13.66(07)$ & $13.59(07)$ & 13.73(07) & 14.63(06) \\
\hline 58395 & 211 & $13.63(09)$ & $13.77(07)$ & $13.71(07)$ & $13.84(07)$ & $14.82(07)$ \\
\hline 58397 & 213 & $13.72(09)$ & $13.92(07)$ & $13.79(07)$ & $13.83(07)$ & 14.76(07) \\
\hline 58400 & 216 & 13.54(09) & $13.74(08)$ & $13.59(07)$ & $13.62(08)$ & $14.33(07)$ \\
\hline
\end{tabular}

${ }^{a}$ Days relative to the initial optical outburst (UT March $06.582018=$ MJD 58184.08).

Note: Uncertainties, in units of $0.01 \mathrm{mag}$, are $1 \sigma$. 
Hanna Sai et al.

Table A3 - continued A table continued from the previous one.

\begin{tabular}{|c|c|c|c|c|c|c|}
\hline MJD & Phase $^{a}$ & uvw2 (mag) & uvm2 (mag) & uvw1 (mag) & UVOT u (mag) & UVOT v (mag) \\
\hline 58402 & 218 & 13.54(09) & $13.58(08)$ & $13.40(07)$ & $13.41(07)$ & 14.11(07) \\
\hline 58404 & 220 & $13.46(09)$ & $13.60(07)$ & $13.43(07)$ & $13.39(07)$ & $14.07(06)$ \\
\hline 58407 & 223 & 13.66(09) & $13.75(07)$ & $13.65(07)$ & $13.61(07)$ & $14.29(06)$ \\
\hline 58408 & 224 & $13.62(09)$ & $13.73(07)$ & $13.54(07)$ & $13.62(07)$ & $14.24(06)$ \\
\hline 58410 & 226 & 13.68(09) & $13.77(07)$ & $13.62(07)$ & $13.56(07)$ & $14.35(06)$ \\
\hline 58412 & 228 & 13.74(09) & $13.85(07)$ & $13.69(07)$ & $13.63(07)$ & $14.42(06)$ \\
\hline 58417 & 233 & 13.94(09) & $14.06(07)$ & 13.91(07) & $13.91(07)$ & $14.70(06)$ \\
\hline 58420 & 236 & $14.09(09)$ & $14.15(07)$ & $14.00(07)$ & $14.05(07)$ & $14.73(06)$ \\
\hline 58422 & 238 & $14.13(09)$ & $14.21(07)$ & $14.11(07)$ & . & $\ldots$ \\
\hline 58425 & 241 & $14.28(09)$ & $14.38(08)$ & $14.26(07)$ & $14.26(07)$ & $15.01(07)$ \\
\hline 58428 & 244 & $14.35(09)$ & $14.46(07)$ & $14.36(07)$ & $14.35(07)$ & $15.09(06)$ \\
\hline 58431 & 247 & $14.59(09)$ & $14.62(08)$ & $14.48(07)$ & $14.57(07)$ & $15.23(07)$ \\
\hline 58433 & 249 & $14.63(10)$ & $14.78(09)$ & $14.49(08)$ & $14.43(06)$ & 15.31(09) \\
\hline 58434 & 250 & $14.83(09)$ & $14.92(08)$ & $14.77(07)$ & $14.70(07)$ & $15.58(07)$ \\
\hline 58437 & 253 & $14.87(09)$ & $15.04(08)$ & $14.91(07)$ & $14.77(07)$ & $15.53(07)$ \\
\hline 58439 & 255 & $15.14(09)$ & $15.28(08)$ & $14.94(07)$ & $14.86(07)$ & $15.87(07)$ \\
\hline 58440 & 256 & $15.21(09)$ & $15.43(08)$ & $15.25(08)$ & $15.21(07)$ & $15.98(07)$ \\
\hline 58546 & 362 & $18.99(15)$ & $18.81(16)$ & $18.26(13)$ & $17.89(12)$ & $18.15(15)$ \\
\hline 58552 & 368 & $17.21(10)$ & $17.13(10)$ & $16.57(09)$ & $\ldots$ & $\ldots$ \\
\hline 58559 & 375 & 13.76(09) & $13.82(07)$ & $13.68(07)$ & $13.69(07)$ & $14.31(06)$ \\
\hline 58566 & 382 & 13.44(09) & $13.60(07)$ & 13.33(07) & 13.41(07) & $14.01(06)$ \\
\hline 58576 & 392 & $\ldots$ & $\cdots$ & $\ldots$ & $13.68(07)$ & $\ldots$ \\
\hline 58591 & 407 & $\ldots$ & $14.58(07)$ & $\ldots$ & $\ldots$ & $\ldots$ \\
\hline 58602 & 418 & $15.54(11)$ & $\ldots$ & $\ldots$ & $\ldots$ & $\ldots$ \\
\hline 58607 & 423 & $\ldots$ & $\ldots$ & $16.35(07)$ & $\ldots$ & $\ldots$ \\
\hline 58612 & 428 & $\ldots$ & $\ldots$ & $17.65(10)$ & $\ldots$ & $\ldots$ \\
\hline 58614 & 430 & $18.54(13)$ & $\ldots$ & $\ldots$ & $\ldots$ & $\ldots$ \\
\hline 58615 & 431 & $\ldots$ & $\ldots$ & $18.01(20)$ & $17.57(15)$ & $\ldots$ \\
\hline 58616 & 432 & $18.88(19)$ & $18.28(19)$ & 17.97(12) & 17.67(09) & $\ldots$ \\
\hline 58620 & 436 & $\ldots$ & $\ldots$ & $18.21(11)$ & $\ldots$ & $\ldots$ \\
\hline 58622 & 438 & $\ldots$ & $18.75(13)$ & $\ldots$ & $\ldots$ & $\ldots$ \\
\hline 58626 & 442 & $18.78(13)$ & $\ldots$ & $\ldots$ & $\ldots$ & $\ldots$ \\
\hline
\end{tabular}

${ }^{a}$ Days relative to the initial optical outburst (UT March 06.58 $2018=$ MJD 58184.08).

Note: Uncertainties, in units of $0.01 \mathrm{mag}$, are $1 \sigma$. 
Table A4. Log of Spectroscopic Observations of MAXI J1820+070

\begin{tabular}{|c|c|c|c|c|}
\hline MJD & Phase $^{a}$ & Range $(\AA)$ & Disp. $(\AA /$ pix $)$ & Telescope+ + Inst. \\
\hline 58195.93 & 11.85 & $3483-8745$ & 2.85 & LJT+YFOSC \\
\hline 58196.86 & 12.78 & $3971-8822$ & 2.78 & $\mathrm{XLT}+\mathrm{BFOSC}$ \\
\hline 58196.93 & 12.85 & $3493-8750$ & 2.85 & LJT+YFOSC \\
\hline 58198.92 & 14.84 & $3483-8742$ & 2.85 & LJT+YFOSC \\
\hline 58201.81 & 17.73 & $3967-8823$ & 2.78 & $\mathrm{XLT}+\mathrm{BFOSC}$ \\
\hline 58201.92 & 17.84 & $3491-8749$ & 2.85 & LJT+YFOSC \\
\hline 58207.83 & 23.75 & $3489-8743$ & 2.85 & $\mathrm{LJT}+\mathrm{YFOSC}$ \\
\hline 58209.85 & 25.77 & $3489-8744$ & 2.85 & LJT+YFOSC \\
\hline 58211.82 & 27.74 & $3489-8743$ & 2.85 & LJT+YFOSC \\
\hline 58212.83 & 28.75 & $3486-8743$ & 2.85 & $\mathrm{LJT}+\mathrm{YFOSC}$ \\
\hline 58223.86 & 39.78 & $3488-8745$ & 2.85 & LJT+YFOSC \\
\hline 58224.76 & 40.68 & $3971-8828$ & 2.78 & XLT+BFOSC \\
\hline 58224.76 & 40.68 & $3971-8828$ & 2.78 & $\mathrm{XLT}+\mathrm{BFOSC}$ \\
\hline 58226.82 & 42.74 & $3980-8828$ & 2.78 & $\mathrm{XLT}+\mathrm{BFOSC}$ \\
\hline 58226.83 & 42.75 & $3980-8827$ & 2.78 & $\mathrm{XLT}+\mathrm{BFOSC}$ \\
\hline 58227.78 & 43.70 & $3972-8826$ & 2.78 & $\mathrm{XLT}+\mathrm{BFOSC}$ \\
\hline 58227.80 & 43.72 & $3972-8826$ & 2.78 & $\mathrm{XLT}+\mathrm{BFOSC}$ \\
\hline 58227.82 & 43.74 & $3972-8826$ & 2.78 & XLT+BFOSC \\
\hline 58236.74 & 52.66 & $3967-8823$ & 2.78 & $\mathrm{XLT}+\mathrm{BFOSC}$ \\
\hline 58236.76 & 52.68 & $3969-8824$ & 2.78 & $\mathrm{XLT}+\mathrm{BFOSC}$ \\
\hline 58245.81 & 61.73 & $3974-8827$ & 2.78 & $\mathrm{XLT}+\mathrm{BFOSC}$ \\
\hline 58246.82 & 62.74 & $4004-8872$ & 2.78 & $\mathrm{XLT}+\mathrm{BFOSC}$ \\
\hline 58282.75 & 98.67 & $3979-8830$ & 2.78 & $\mathrm{XLT}+\mathrm{BFOSC}$ \\
\hline 58296.61 & 112.53 & $3971-8822$ & 2.78 & XLT+BFOSC \\
\hline 58296.63 & 112.55 & $3973-8824$ & 2.78 & $\mathrm{XLT}+\mathrm{BFOSC}$ \\
\hline 58297.66 & 113.59 & $3969-8821$ & 2.78 & $\mathrm{XLT}+\mathrm{BFOSC}$ \\
\hline 58297.68 & 113.60 & $3969-8821$ & 2.78 & XLT+BFOSC \\
\hline 58297.69 & 113.61 & $3970-8822$ & 2.78 & $\mathrm{XLT}+\mathrm{BFOSC}$ \\
\hline 58297.71 & 113.63 & $3969-8821$ & 2.78 & $\mathrm{XLT}+\mathrm{BFOSC}$ \\
\hline 58297.72 & 113.64 & $3970-8822$ & 2.78 & XLT+BFOSC \\
\hline 58297.74 & 113.66 & $3970-8822$ & 2.78 & $\mathrm{XLT}+\mathrm{BFOSC}$ \\
\hline 58386.55 & 202.47 & $3850-8700$ & 2.78 & $\mathrm{XLT}+\mathrm{BFOSC}$ \\
\hline 58390.46 & 206.38 & $3854-8701$ & 2.78 & $\mathrm{XLT}+\mathrm{BFOSC}$ \\
\hline 58390.48 & 206.40 & $3854-8701$ & 2.78 & $\mathrm{XLT}+\mathrm{BFOSC}$ \\
\hline 58390.50 & 206.42 & $3854-8701$ & 2.78 & $\mathrm{XLT}+\mathrm{BFOSC}$ \\
\hline 58392.46 & 208.38 & $3852-8698$ & 2.78 & $\mathrm{XLT}+\mathrm{BFOSC}$ \\
\hline 58392.48 & 208.40 & $3852-8698$ & 2.78 & $\mathrm{XLT}+\mathrm{BFOSC}$ \\
\hline 58392.50 & 208.42 & $3852-8698$ & 2.78 & XLT+BFOSC \\
\hline 58395.46 & 211.38 & $3970-8697$ & 2.78 & XLT+BFOSC \\
\hline 58395.48 & 211.40 & $3970-8696$ & 2.78 & $\mathrm{XLT}+\mathrm{BFOSC}$ \\
\hline 58396.49 & 212.41 & 3973-8699 & 2.78 & $\mathrm{XLT}+\mathrm{BFOSC}$ \\
\hline 58396.50 & 212.42 & 3973-8699 & 2.78 & $\mathrm{XLT}+\mathrm{BFOSC}$ \\
\hline 58399.44 & 215.36 & $3970-8696$ & 2.78 & $\mathrm{XLT}+\mathrm{BFOSC}$ \\
\hline 58399.46 & 215.38 & $3970-8696$ & 2.78 & $\mathrm{XLT}+\mathrm{BFOSC}$ \\
\hline 58400.45 & 216.37 & $3973-8699$ & 2.78 & $\mathrm{XLT}+\mathrm{BFOSC}$ \\
\hline 58400.47 & 216.39 & $3973-8699$ & 2.78 & $\mathrm{XLT}+\mathrm{BFOSC}$ \\
\hline 58401.45 & 217.37 & $3976-8701$ & 2.78 & XLT+BFOSC \\
\hline 58401.47 & 217.39 & $3976-8701$ & 2.78 & $\mathrm{XLT}+\mathrm{BFOSC}$ \\
\hline 58404.48 & 220.40 & $5139-8776$ & 4.8 & $\mathrm{XLT}+\mathrm{OMR}$ \\
\hline 58409.44 & 225.36 & $3762-8689$ & 4.8 & $\mathrm{XLT}+\mathrm{OMR}$ \\
\hline 58421.49 & 237.41 & $3976-8698$ & 2.78 & XLT+BFOSC \\
\hline 58431.41 & 247.33 & $3856-8699$ & 2.78 & XLT+BFOSC \\
\hline 58435.44 & 251.36 & $3855-8701$ & 2.78 & XLT+BFOSC \\
\hline 58441.48 & 257.40 & $3486-8747$ & 2.85 & LJT+YFOSC \\
\hline 58444.42 & 260.34 & $3857-8704$ & 2.78 & $\mathrm{XLT}+\mathrm{BFOSC}$ \\
\hline 58446.42 & 262.34 & $3853-8698$ & 2.78 & $\mathrm{XLT}+\mathrm{BFOSC}$ \\
\hline 58447.42 & 263.34 & $3855-8699$ & 2.78 & $\mathrm{XLT}+\mathrm{BFOSC}$ \\
\hline 58555.82 & 371.74 & $4358-8710$ & 2.78 & $\mathrm{XLT}+\mathrm{BFOSC}$ \\
\hline 58559.81 & 375.73 & $3860-8825$ & 2.78 & $\mathrm{XLT}+\mathrm{BFOSC}$ \\
\hline 58559.86 & 375.78 & $3862-8825$ & 2.78 & $\mathrm{XLT}+\mathrm{BFOSC}$ \\
\hline 58564.81 & 380.73 & $3863-8826$ & 2.78 & $\mathrm{XLT}+\mathrm{BFOSC}$ \\
\hline 58564.84 & 380.76 & $3866-8829$ & 2.78 & $\mathrm{XLT}+\mathrm{BFOSC}$ \\
\hline 58566.85 & 382.77 & $3861-8826$ & 2.78 & $\mathrm{XLT}+\mathrm{BFOSC}$ \\
\hline 58568.83 & 384.75 & $3863-8827$ & 2.78 & $\mathrm{XLT}+\mathrm{BFOSC}$ \\
\hline 58568.85 & 384.77 & $3864-8828$ & 2.78 & $\mathrm{XLT}+\mathrm{BFOSC}$ \\
\hline 58568.86 & 384.78 & $3864-8828$ & 2.78 & $\mathrm{XLT}+\mathrm{BFOSC}$ \\
\hline
\end{tabular}

